\title{
THE EXISTENCE OF A SOLUTION AND A NUMERICAL METHOD FOR THE TIMOSHENKO NONLINEAR WAVE SYSTEM
}

\author{
Jemal PeRADZE ${ }^{1}$
}

\begin{abstract}
The initial boundary value problem for a beam is considered in the Timoshenko model. Assuming the analyticity of the initial conditions, it is proved that the problem is solvable throughout the time interval. After that, a numerical algorithm, consisting of three steps, is constructed. The solution is approximated with respect to the spatial and time variables using the Galerkin method and a Crank-Nicholson type scheme. The system of equations obtained by discretization is solved by a version of the Picard iteration method. The accuracy of the proposed algorithm is investigated.
\end{abstract}

Mathematics Subject Classification. 35Q, 65M.

Received: July 24, 2003.

\section{PART I. The PROBlem AND ITS SOLVABILITY}

\section{Formulation of the PROBlem}

This paper deals with the problem of geometrically nonlinear vibration of a beam. To this end, we use the well-known Timoshenko model which is a theory of the second generation. As compared with the classical Kirchhoff-Love theory, the Timoshenko model allows us to take into account deformation produced by cross force and rotary inertia, which is important for a lot of problems.

Thus, let us consider the system of equations

$$
\begin{array}{ll}
\frac{\partial^{2} w}{\partial t^{2}}(x, t)=\left(c d-a+b \int_{0}^{1}\left(\frac{\partial w}{\partial x}(x, t)\right)^{2} \mathrm{~d} x\right) \frac{\partial^{2} w}{\partial x^{2}}(x, t)-c d \frac{\partial \psi}{\partial x}(x, t), \\
\frac{\partial^{2} \psi}{\partial t^{2}}(x, t)=c \frac{\partial^{2} \psi}{\partial x^{2}}(x, t)-c^{2} d\left(\psi(x, t)-\frac{\partial w}{\partial x}(x, t)\right), & 0<x<1, \quad 0<t \leq T,
\end{array}
$$

with the initial and boundary conditions

$$
\begin{gathered}
\frac{\partial^{l} w}{\partial t^{l}}(x, 0)=w^{l}(x), \quad \frac{\partial^{l} \psi}{\partial t^{l}}(x, 0)=\psi^{l}(x), \quad l=0,1, \\
w(0, t)=w(1, t)=0, \frac{\partial \psi}{\partial x}(0, t)=\frac{\partial \psi}{\partial x}(1, t)=0, \quad 0 \leq x \leq 1, \quad 0 \leq t \leq T .
\end{gathered}
$$

Keywords and phrases. Timoshenko nonlinear system, beam, Galerkin method, Crank-Nicholson scheme, Picard process.

${ }^{1}$ Department of Applied Mathematics and Computer Sciences of Tbilisi State University, Tbilisi, 380043, R. of Georgia. e-mail: j-peradze@yahoo.com 
System (1.1) describing the dynamic state of a beam was proposed in the work of Hirschhorn and Reiss [2]. The boundary conditions (1.3) correspond to the case of a beam with hinged ends. Linearizing (1.1), we obtain the system for the linear Timoshenko beam [3].

The functions $w(x, t)$ and $\psi(x, t)$ are respectively transverse deflection of the beam centerline and rotary displacement of the beam cross-section.

The constants

$$
a, b, c, d>0 \quad \text { and } \quad c d-a>0 .
$$

Here

$$
a=\frac{A e S}{M_{1}}, \quad b=\frac{A e^{2}}{2 M_{1}}, \quad c=\frac{A e^{2}}{M_{2}}, \quad d=\frac{G M_{2}}{E M_{1}},
$$

$E$ is Young's modulus, $G$ is the shear modulus, $A$ is the cross-section area, $e$ is the beam length, $M_{1}$ is the moment of inertia of the cross-section about the axis perpendicular to the beam centerline, $M_{2}$ is the polar moment of inertia of the cross-section, and $S$ is the end shortening of the beam.

By virtue of (1.5) we come to a conclusion that the second relation in (1.4) is a natural requirement for moderately compressed slender beams since it is equivalent to the condition $S<\frac{e G}{E}$.

The question of the existence of a solution of problem (1.1)-(1.3) was posed for the first time by Tucsnak in [3] who proved that the problem is solvable locally with respect to time if the functions $w^{l}(x)$ and $\psi^{l}(x)$, $l=0,1$, are differentiable a finite number of times.

Here it is assumed that

$$
\begin{aligned}
& w^{l}(x) \text { and } \psi^{l}(x) \text { are analytic functions of the types } \\
& w^{l}(x)=\sum_{i=1}^{\infty} a_{i}^{l} \sin i \pi x, \quad \psi^{l}(x)=\frac{b_{0}^{l}}{\sqrt{2}}+\sum_{j=1}^{\infty} b_{j}^{l} \cos j \pi x, \quad l=0,1 .
\end{aligned}
$$

\section{Existence of A solution}

To prove the existence of a global solution of problem (1.1)-(1.3), we will use the approach by means of which Bernstein obtained, in [1], the solvability of the equation

$$
\frac{\partial^{2} u}{\partial t^{2}}=\varphi\left(\int_{0}^{l}\left(\frac{\partial u}{\partial x}\right)^{2} \mathrm{~d} x\right) \frac{\partial^{2} u}{\partial x^{2}}
$$

\subsection{The infinite system}

Let us consider the series

$$
w(x, t)=\sum_{i=1}^{\infty} w_{i}(t) \sin i \pi x, \psi(x, t)=\frac{\psi_{0}(t)}{\sqrt{2}}+\sum_{j=1}^{\infty} \psi_{j}(t) \cos j \pi x,
$$

where the coefficients $w_{i}(t)$ and $\psi_{j}(t)$ are defined by the system of ordinary differential equations

$$
\begin{aligned}
& \frac{\mathrm{d}^{2} w_{i}}{\mathrm{~d} t^{2}}(t)+\left(c d-a+\frac{b}{2} \pi^{2} \sum_{k=1}^{\infty} k^{2} w_{k}^{2}(t)\right) \pi^{2} i^{2} w_{i}(t)-c d \pi i \psi_{i}(t)=0, \\
& \frac{\mathrm{d}^{2} \psi_{j}}{\mathrm{~d} t^{2}}(t)+c \pi^{2} j^{2} \psi_{j}(t)+c^{2} d\left(\psi_{j}(t)-\pi j w_{j}(t)\right)=0, \quad 0<t \leq T, \quad\left(w_{0}(t)=0\right),
\end{aligned}
$$

with the initial condition

$$
\frac{\mathrm{d}^{l} w_{i}}{\mathrm{~d} t^{l}}(0)=a_{i}^{l}, \quad \frac{\mathrm{d}^{l} \psi_{j}}{\mathrm{~d} t^{l}}(0)=b_{j}^{l}, \quad l=0,1, i=1,2, \ldots, j=0,1, \ldots
$$




\subsection{The finite system}

Let us write a finite-dimensional analogue of problem (2.2), (2.3). Assume that for $n \geq 1$,

$$
w_{n}(x, t)=\sum_{i=1}^{n} w_{n i}(t) \sin i \pi x, \quad \psi_{n}(x, t)=\frac{\psi_{n 0}(t)}{\sqrt{2}}+\sum_{J=1}^{n} \psi_{n j}(t) \cos j \pi x,
$$

where for $0<t \leq T$

$$
\begin{aligned}
& \frac{\mathrm{d}^{2} w_{n i}}{\mathrm{~d} t^{2}}(t)+\left(c d-a+\frac{b}{2} \pi^{2} \sum_{k=1}^{n} k^{2} w_{n k}^{2}(t)\right) \pi^{2} i^{2} w_{n i}(t)-c d \pi i \psi_{n i}(t)=0, \\
& \frac{\mathrm{d}^{2} \psi_{n j}}{\mathrm{~d} t^{2}}(t)+c \pi^{2} j^{2} \psi_{n j}(t)+c^{2} d\left(\psi_{n j}(t)-\pi j w_{n j}(t)\right)=0, \quad\left(w_{n 0}(t)=0\right),
\end{aligned}
$$

and

$$
\frac{\mathrm{d}^{l} w_{n i}}{\mathrm{~d} t^{l}}(0)=a_{i}^{l}, \quad \frac{\mathrm{d}^{l} \psi_{n j}}{\mathrm{~d} t^{l}}(0)=b_{j}^{l}, \quad l=0,1, i=1,2, \ldots, n, j=0,1, \ldots, n .
$$

\subsection{Solvability of the finite system}

Lemma 1. System $(2.5,2.6)$ has a solution for $0<t \leq T$.

Proof. Multiply the first equation of system (2.5) by $2 \frac{\mathrm{d} w_{n i}}{\mathrm{~d} t}(t)$ and the second by $2 \frac{1}{c} \frac{\mathrm{d} \psi_{n j}}{\mathrm{~d} t}(t)$. After that sum each of them over $i=1,2, \ldots, n$ and $j=0,1, \ldots, n$, respectively, and add the obtained relations. This results in

$$
\frac{\mathrm{d} E_{n}}{\mathrm{~d} t}=0
$$

where

$$
\begin{aligned}
E_{n}(t)=\sum_{i=1}^{n}\left[\left(\frac{\mathrm{d} w_{n i}}{\mathrm{~d} t}(t)\right)^{2}+\pi^{2} i^{2} \psi_{n i}^{2}(t)\right]+\sum_{j=0}^{n}\left[\frac{1}{c}\left(\frac{\mathrm{d} \psi_{n j}}{\mathrm{~d} t}(t)\right)^{2}+c d\left(\psi_{n j}(t)-\pi j w_{n j}(t)\right)^{2}\right] & \\
+\frac{1}{b}\left(-a+\frac{b}{2} \pi^{2} \sum_{i=1}^{n} i^{2} w_{n i}^{2}(t)\right)^{2}, & \left(w_{n 0}(t)=0\right) .
\end{aligned}
$$

By $(2.7,2.8)$ and also by $(1.6,2.6)$, we conclude that $E_{n}(t)=E_{n}(0), \lim _{n \rightarrow \infty} E_{n}(0)=E_{0}$ and $E_{n}(0) \leq E_{0}$, where

$$
\begin{aligned}
E_{0}=2 \int_{0}^{1}\left[\left(w^{1}(x)\right)^{2}+c d\left(\psi^{0}(x)-\frac{\mathrm{d} w^{0}}{\mathrm{~d} x}(x)\right)^{2}+\frac{1}{2 b}\left(-a+b \int_{0}^{1}\left(\frac{\mathrm{d} w^{0}}{\mathrm{~d} x}(x)\right)^{2} \mathrm{~d} x\right)^{2}\right. \\
\left.+\frac{1}{c}\left(\psi^{1}(x)\right)^{2}+\left(\frac{\mathrm{d} \psi^{0}}{\mathrm{~d} x}(x)\right)^{2}\right] \mathrm{d} x .
\end{aligned}
$$

Therefore

$$
E_{n}(t) \leq E_{0}
$$

i.e. $E_{n}(t)$ is bounded by the constant that does not depend on $n$ and $t$.

From the Cauchy theorem follows the existence of twice differentiable functions $w_{n i}(t)$ and $\psi_{n j}(t)$ which satisfy the system of equations (2.5) for $0<t \leq T$ and the initial condition (2.6).

The latter fact and (2.5) imply the following property, which we will use below. 
Corollary 1. For $0<t<T$ the functions $w_{n i}(t)$ and $\psi_{n j}(t)$ have continuous derivatives up to fourth order inclusive, $i=1,2, \ldots, n, \quad j=0,1, \ldots, n$.

\subsection{Solvability of the infinite system}

By virtue of (2.9), the sequences of functions $\left(\frac{\mathrm{d}^{l} w_{n i}}{\mathrm{~d} t^{l}}(t)\right)_{n=1}^{\infty}$ and $\left(\frac{\mathrm{d}^{l} \psi_{n j}}{\mathrm{~d} t^{l}}(t)\right)_{n=0}^{\infty}, l=0,1$, are bounded for each fixed $i$ and $j$. Hence, when passing, if required, to subsequences, we see that, on $[0, T]$, the sequences $w_{n i}(t)$ and $\psi_{n j}(t)$ uniformly tend to some continuous functions as $n \rightarrow \infty$, which in order to avoid the introduction of new notations, are denoted by $w_{i}(t)$ and $\psi_{j}(t)$, respectively. Let us show that these functions satisfy system $(2.2,2.3)$.

From (1.6) it follows that there exist constants $Q>0$ and $R>1$ such that [1]

$$
i^{5-2 l}\left(a_{i}^{l}\right)^{2}<\frac{Q}{R^{i}}, \quad j^{5-2 l}\left(b_{j-1}^{l}\right)^{2}<\frac{Q}{R^{j}}, \quad l=0,1, i, j=1,2, \ldots
$$

Next, note that if, for some $t$, the finite series

$$
r_{n}(t, R)=\sum_{i=1}^{n} i R^{i}\left[\pi^{2} i^{2} w_{n i}^{2}(t)+\Delta_{n}^{-1}(t)\left(\frac{\mathrm{d} w_{n i}}{\mathrm{~d} t}(t)\right)^{2}+\pi^{2} i^{2} \psi_{n i}^{2}(t)+\frac{1}{c}\left(\frac{\mathrm{d} \psi_{n i}}{\mathrm{~d} t}(t)\right)^{2}+c d \psi_{n i}^{2}(t)\right],
$$

where

$$
\Delta_{n}(t)=c d-a+\frac{b}{2} \pi^{2} \sum_{j=1}^{n} j^{2} w_{n j}^{2}(t)
$$

has the boundary not depending on $n$, then the same value is the boundary for the series

$$
r(t, R)=\sum_{i=1}^{\infty} i R^{i}\left[\pi^{2} i^{2} w_{i}^{2}(t)+\Delta^{-1}(t)\left(\frac{\mathrm{d} w_{i}}{\mathrm{~d} t}(t)\right)^{2}+\pi^{2} i^{2} \psi_{i}^{2}(t)+\frac{1}{c}\left(\frac{\mathrm{d} \psi_{i}}{\mathrm{~d} t}(t)\right)^{2}+c d \psi_{i}^{2}(t)\right],
$$

where

$$
\begin{gathered}
\Delta(t)=c d-a+\frac{b}{2} \pi^{2} \sum_{j=1}^{\infty} j^{2} w_{j}^{2}(t), \\
\frac{\mathrm{d}^{l} w_{i}}{\mathrm{~d} t^{l}}(t)=\lim _{n \rightarrow \infty} \frac{\mathrm{d}^{l} w_{n i}}{\mathrm{~d} t^{l}}(t), \quad \frac{\mathrm{d}^{l} \psi_{i}}{\mathrm{~d} t^{l}}(t)=\lim _{n \rightarrow \infty} \frac{\mathrm{d}^{l} \psi_{n i}}{\mathrm{~d} t^{l}}(t), \quad l=0,1 .
\end{gathered}
$$

Hence, for brevity, we will consider the series $r(t, R)$ directly.

Lemma 2. If the series $r(t, R)$ converges for some $t=t_{0}$, then the series $r(t, 1)$ converges uniformly for $\left|t-t_{0}\right| \leq \frac{1}{\mu} \ln R$, where $\mu$ is some constant not depending on $t$.

Proof. Consider the function

$$
F_{i}(t)=\pi^{2} i^{2} w_{i}^{2}(t)+\left(\frac{\mathrm{d} w_{i}}{\mathrm{~d} t}(t)\right)^{2}+\pi^{2} i^{2} \psi_{i}^{2}(t)+\frac{1}{c}\left(\frac{\mathrm{d} \psi_{i}}{\mathrm{~d} t}(t)\right)^{2}+c d \psi_{i}^{2}(t) .
$$

From (2.2) it follows that

$$
\frac{\mathrm{d} F_{i}}{\mathrm{~d} t}(t)=2 \pi^{2} i^{2} \frac{\mathrm{d} w_{i}}{\mathrm{~d} t}(t) w_{i}(t)(1-\Delta(t))+2 c d \pi i \frac{\mathrm{d}}{\mathrm{d} t}\left(w_{i}(t) \psi_{i}(t)\right) .
$$


Based on $(2.8,2.9)$, we conclude that $\Delta(t)$ is uniformly bounded. Hence $\left|\frac{\mathrm{d} F_{i}}{\mathrm{~d} t}(t)\right| \leq \mu i F_{i}(t)$, where $\mu$ is some constant not depending on $t$ and therefore if $\exp \left(\mu\left|t-t_{0}\right|\right) \leq R$, then

$$
F_{i}(t) \leq F_{i}\left(t_{0}\right) \exp \left(\mu i\left|t-t_{0}\right|\right) \leq F_{i}\left(t_{0}\right) R^{i}
$$

Now, taking (2.12) into account, we obtain the validity of the lemma.

From Lemma 2 and the fact that $r(0, R)$ converges by virtue of $(2.10)$ it follows in particular that the function $r(t, 1)$ is finite and continuous for $t \leq \frac{1}{\mu} \ln R$.

Lemma 3. There exists no finite value of $t$ for which the series $r(t, R)$ is not convergent.

Proof. After differentiating (2.12) and applying (2.2), we find

$$
\frac{\mathrm{d} r}{\mathrm{~d} t}(t, R)=\sum_{i=1}^{\infty} i R^{i}\left[-b \pi^{2} \Delta^{-2}(t)\left(\frac{\mathrm{d} w_{i}}{\mathrm{~d} t}(t)\right)^{2} \sum_{j=1}^{\infty} j^{2} \frac{\mathrm{d} w_{j}}{\mathrm{~d} t}(t) w_{j}(t)+2 c d \pi i\left(\Delta^{-1}(t) \frac{\mathrm{d} w_{i}}{\mathrm{~d} t}(t) \psi_{i}(t)+w_{i}(t) \frac{\mathrm{d} \psi_{i}}{\mathrm{~d} t}(t)\right)\right]
$$

This and (2.12) imply

$$
\left|\frac{\mathrm{d} r}{\mathrm{~d} t}(t, R)\right| \leq r(t, R)\left(a_{1} r(t, 1)+a_{2}\right)
$$

where

$$
a_{1}=\pi \frac{b}{2}(c d-a)^{-\frac{1}{2}}, \quad a_{2}=c d \max ^{\frac{1}{2}}\left((c d-a)^{-1}, c\right) .
$$

Assume now that $r(t, R)$ is finite for some $t=t_{1}$. Then $r\left(t_{1}, 1\right)$ is finite, too.

Denote

$$
g(t)=1+\frac{a_{2}}{a_{1}} r^{-1}(t, 1), \quad p(t)=\left[1-g\left(t_{1}\right) \exp \left(a_{2}\left(t_{1}-t\right)\right)\right]^{-1} .
$$

For $R=1$, from (2.13) we obtain $|d \ln g(t)|<a_{2} \mathrm{~d} t$ and therefore if

$$
0<t-t_{1}<\frac{1}{a_{2}} \ln g\left(t_{1}\right)
$$

then $r(t, 1)<-\frac{a_{2}}{a_{1}} p(t)$. The latter inequality together with $(2.13)$ gives $\left|\frac{\mathrm{d} \ln r}{\mathrm{~d} t}(t, R)\right|<a_{2}(1-p(t))$. Therefore $\ln \left|\frac{r(t, R)}{r\left(t_{1}, R\right)}\right| \leq \ln \left[\left(1-g\left(t_{1}\right)\right) p(t)\right]$.

Hence we conclude that when (2.14) is fulfilled, the estimate

$$
r(t, R) \leq r\left(t_{1}, R\right) \exp \left(a_{2}\left(t-t_{1}\right)\right) \frac{1}{1+\frac{a_{1}}{a_{2}} r\left(t_{1}, 1\right)\left[1-\exp \left(a_{2}\left(t-t_{1}\right)\right)\right]}
$$

is true.

Further, if the series $r(t, R)$ converges for all positive $t<t_{0}$, then by Lemma 2 the series $r(t, 1)$ converges uniformly when $t_{0}-\frac{1}{\mu} \ln R \leq t \leq t_{0}$. Therefore there exists a finite value $H>0$ such that $r(t, 1)<H$ in this interval. In view of inequalities $(2.14),(2.15)$ we conclude that if $t_{1}<t_{0}$ is chosen so close to $t_{0}$ that $t_{0}-t_{1}<\varepsilon$, where $\varepsilon=\frac{1}{a_{2}} \ln \left(1+\frac{a_{2}}{a_{1}} H^{-1}\right)$, then $r\left(t_{0}, R\right)$ is also finite. Thus there exists no $t_{0}$ such that the series $r(t, R)$ is convergent for all $t<t_{0}$ and is not convergent for $t=t_{0}+\frac{1}{2} \varepsilon$.

This lemma and the arguments given in its proof imply the following propositions that we will use later on.

Corollary 2. The series $\sum_{i=1}^{\infty} i^{l}\left|w_{i}(t)\right|$ and $\sum_{j=1}^{\infty} j^{l}\left|\psi_{j}(t)\right|$ converge, $l=-2,-1, \ldots, 2,0 \leq t \leq T$.

Corollary 3. The series $r_{n}(t, R)$ and $r(t, R)$ are uniformly bounded, the former with respect to $n$ and $t$, and the latter with respect to $t, n=1,2, \ldots, 0<t \leq T$. 
Each pair of equations (2.5) with the boundary conditions (2.6) is equivalent to the equations

$$
\begin{aligned}
& w_{n i}(t)=a_{i}^{0}+a_{i}^{1} t+\int_{0}^{t}(\tau-t)\left[\left(c d-a+\frac{b}{2} \pi^{2} \sum_{k=1}^{\infty} k^{2} w_{n k}^{2}(\tau)\right) \pi^{2} i^{2} w_{n i}(\tau)-c d \pi i \psi_{n i}(\tau)\right] \mathrm{d} \tau, \\
& \psi_{n j}(t)=b_{j}^{0}+b_{j}^{1} t+\int_{0}^{t}(\tau-t)\left[c \pi^{2} j^{2} \psi_{n j}(\tau)+c^{2} d\left(\psi_{n j}(\tau)-\pi j w_{n j}(\tau)\right)\right] \mathrm{d} \tau, \quad\left(w_{n k}(t)=0 \text { for } k=0 \text { and } k>n\right) .
\end{aligned}
$$

Since all functions $w_{n i}(t)$ and $\psi_{n j}(t)$ satisfy these equations for $i=1,2, \ldots, n, j=0,1, \ldots, n$, and for any $0 \leq t \leq T$ and, moreover, for given $i$ and $j$ the functions $w_{n i}(t)$ and $\psi_{n j}(t)$ tend uniformly, as $n \rightarrow \infty$, to $w_{i}(t)$ and $\psi_{j}(t)$, for which (2.3) is fulfilled, while, as follows from (2.9) and (2.15), $\sum_{k=1}^{n} k^{2} w_{n k}^{2}(t)$ tends uniformly to $\sum_{k=1}^{\infty} k^{2} w_{k}^{2}(t)$, we see that $w_{i}(t)$ and $\psi_{j}(t)$ satisfy the same equations (2.16) for any $i$ and $j$.

Thus for $0<t \leq T$ the functions $w_{i}(t)$ and $\psi_{j}(t)$ are twice differentiable and satisfy the system of equations (2.2) and the initial conditions (2.3).

\subsection{Solvability of the initial problem}

Let us introduce the functions

$$
\begin{aligned}
W^{(l)}(x, t) & =-\frac{1}{\pi^{l-1}} \sum_{i=1}^{\infty} \frac{1}{i^{l+1}} w_{i}(t) \frac{\mathrm{d}^{l-1} \sin i \pi x}{\mathrm{~d} x^{l-1}}, \\
\Psi^{(l)}(x, t) & =-\frac{1}{\pi^{l-1}} \sum_{j=1}^{\infty} \frac{1}{j^{l+1}} \psi_{j}(t) \frac{\mathrm{d}^{l-1} \cos j \pi x}{\mathrm{~d} x^{l-1}}, \quad l=1,2 .
\end{aligned}
$$

By virtue of Corollary 2, the series in equalities (2.1) and (2.17) converge absolutely. Hence, after multiplying, for $i, j=1,2, \ldots$, the equations of system $(2.2)$ by $\frac{1}{i^{2}} \sin i \pi x$ and $\frac{1}{j^{2}} \cos j \pi x$, respectively, and summing them, we obtain the absolute convergence of the series

$$
-\sum_{i=1}^{\infty} \frac{1}{i^{2}} \frac{\mathrm{d}^{2} w_{i}}{\mathrm{~d} t^{2}}(t) \sin i \pi x=\frac{\partial^{2} W^{(1)}}{\partial t^{2}}(x, t), \quad-\sum_{j=1}^{\infty} \frac{1}{j^{2}} \frac{\mathrm{d}^{2} \psi_{j}}{\mathrm{~d} t^{2}}(t) \cos j \pi x=\frac{\partial^{2} \Psi^{(1)}}{\partial t^{2}}(x, t) .
$$

Thus the system of equations

$$
\begin{aligned}
& \frac{\partial^{2} W^{(1)}}{\partial t^{2}}(x, t)=\left(c d-a+b \int_{0}^{1}\left(\frac{\partial w}{\partial x}(x, t)\right)^{2} \mathrm{~d} x\right) \pi^{2} w(x, t)-c d \pi \Psi^{(2)}(x, t), \\
& \frac{\partial^{2} \Psi^{(1)}}{\partial t^{2}}(x, t)=c \pi^{2}\left(\psi(x, t)-\frac{\psi_{0}(t)}{\sqrt{2}}\right)-c^{2} d\left(\Psi^{(1)}(x, t)-\pi W^{(2)}(x, t)\right)
\end{aligned}
$$

is fulfilled for $0<x<1$ and $0<t \leq T$.

Let us twice differentiate the equations of system (2.18) with respect to $x$. That this operation is possible follows from Corollary 2. In particular, this gives

$$
\frac{\partial^{2} W^{(1)}}{\partial x^{2}}(x, t)=\pi^{2} w(x, t), \quad \frac{\partial^{2} \Psi^{(1)}}{\partial x^{2}}(x, t)=\pi^{2} \psi(x, t) .
$$

We also take into account the equation $\frac{\mathrm{d}^{2} \psi_{0}}{\mathrm{~d} t^{2}}(t)+c^{2} d \psi_{0}(t)=0$ from system (2.2). This leads us to system (1.1). Thus we have proved. 
Theorem 1. If the initial functions $w^{l}(x)$ and $\psi^{l}(x), l=0,1$, satisfy condition (1.6), then problem (1.1)-(1.3) has a solution, namely, analytic with respect to $0 \leq x \leq 1$ for $0 \leq t \leq T$ functions $w(x, t)$ and $\psi(x, t)$ which are representable by series (2.1).

\section{PART II. NUMERICAL METHOD}

\section{Approximation with Respect to a spatial variable}

\subsection{Galerkin method}

In this stage of construction of the numerical algorithm for problem (1.1)-(1.3) we make use of the Galerkin method. This method has already been used above. Thus we will approach the sought functions $w(x, t)$ and $\psi(x, t)$ by means of sums (2.4). The functions $w_{n i}(t)$ and $\psi_{n j}(t), i=1,2, \ldots, n, j=0,1, \ldots, n$, in (2.4) are solutions of the system of ordinary differential equations (2.5), provided that the values

$$
\frac{\mathrm{d}^{l} w_{n i}}{\mathrm{~d} t^{l}}(0), \frac{\mathrm{d}^{l} \psi_{n j}}{\mathrm{~d} t^{l}}(0) \quad \text { are given, } \quad l=0,1 .
$$

A representation of the initial conditions for $w_{n i}(t)$ and $\psi_{n j}(t)$ in form (3.1) and not by equalities (2.6) means that we may come across the case where the coefficients $a_{i}^{l}$ and $b_{j}^{l}$ in expansions (1.6), which as a rule have to be calculated, are given inexactly.

Let us assume that the analogues of inequalities (2.10)

$$
i^{5-2 l}\left(\frac{\mathrm{d}^{l} w_{n i}}{\mathrm{~d} t^{l}}(0)\right)^{2}<\frac{Q}{R^{i}}, \quad j^{5-2 l}\left(\frac{\mathrm{d}^{l} \psi_{n, j-1}}{\mathrm{~d} t^{l}}(0)\right)^{2}<\frac{Q}{R^{j}}, l=0,1, \quad i=1,2, \ldots, n, \quad j=1,2, \ldots, n+1,
$$

hold, where, for simplicity, we use the same constant $Q>0$ and $R>1$.

\subsection{Matrix notation of the system}

Below we will use the vectors

$$
\boldsymbol{w}_{n}(t)=\left(w_{n i}(t)\right)_{i=1}^{n}, \quad \boldsymbol{\psi}_{n}(t)=\left(\psi_{n j}(t)\right)_{j=0}^{n}
$$

and also $\boldsymbol{w}_{n}^{l}=\left(a_{i}^{l}\right)_{i=1}^{n}, \boldsymbol{\psi}_{n}^{l}=\left(b_{j}^{l}\right)_{j=0}^{n}, l=0,1$.

Let us introduce the scalar product and norms in $R^{n+l}, l=0,1$. If $\alpha$ and $\beta$ are vectors of the same dimension and their $k$ th components are equal to $\alpha_{k}$ and $\beta_{k}$, then the scalar product $(\alpha, \beta)_{n}=\sum_{k} \alpha_{k} \beta_{k}$, where the summation involves all components of $\alpha$ and $\beta$ and the norm $\|\alpha\|_{n}=(\alpha, \alpha)_{n}^{\frac{1}{2}}$. If $D$ is a diagonal matrix with nonnegative elements whose order coincides with the dimension of the vector $\alpha$, then the energy norm $\|\alpha\|_{D}=(D \alpha, \alpha)_{n}^{\frac{1}{2}}$.

In addition to this, let us define two diagonal matrices and one $(n+1) \times n$ matrix

$$
K_{n}=\pi \operatorname{diag}(1,2, \ldots, n), \quad L_{n}=\pi \operatorname{diag}(0,1, \ldots, n), \quad U_{n}=\left(\begin{array}{cccc}
0 & 0 & \ldots & 0 \\
1 & 0 & \ldots & 0 \\
\cdot & \cdot & \cdot & \cdot \\
0 & 0 & \ldots & 1
\end{array}\right)
$$


The above notation allows us to rewrite system (2.5) as

$$
\begin{aligned}
& \frac{\mathrm{d}^{2} \boldsymbol{w}_{n}}{\mathrm{~d} t^{2}}(t)+\left(c d-a+\frac{b}{2}\left\|\boldsymbol{w}_{n}(t)\right\|_{K_{n}^{2}}^{2}\right) K_{n}^{2} \boldsymbol{w}_{n}(t)-c d U_{n}^{\prime} L_{n} \boldsymbol{\psi}_{n}(t)=0, \\
& \frac{\mathrm{d}^{2} \boldsymbol{\psi}_{n}}{\mathrm{~d} t^{2}}(t)+c L_{n}^{2} \boldsymbol{\psi}_{n}(t)+c^{2} d\left(\boldsymbol{\psi}_{n}(t)-L_{n} U_{n} \boldsymbol{w}_{n}(t)\right)=0, \quad 0<t \leq T .
\end{aligned}
$$

Condition (3.1) means that the vectors

$$
\frac{\mathrm{d}^{l} \boldsymbol{w}_{n}}{\mathrm{~d} t^{l}}(0), \frac{\mathrm{d}^{l} \boldsymbol{\psi}_{n}}{\mathrm{~d} t^{l}}(0) \text { are given, } \quad l=0,1
$$

\subsection{Truncation error}

Let us consider the Fourier expansion of the exact solution of problem (1.1)-(1.3)

$$
w(x, t)=\sum_{i=1}^{\infty} w_{i}(t) \sin i \pi x, \quad \psi(x, t)=\frac{\psi_{0}(t)}{\sqrt{2}}+\sum_{j=1}^{\infty} \psi_{j}(t) \cos j \pi x
$$

and construct the vectors $p_{n} \boldsymbol{w}(t)=\left(w_{i}(t)\right)_{i=1}^{n}, p_{n} \boldsymbol{\psi}(t)=\left(\psi_{j}(t)\right)_{j=0}^{n}$.

By (2.2) and (2.3) we obtain the system of equations

$$
\begin{gathered}
\frac{\mathrm{d}^{2} p_{n} \boldsymbol{w}}{\mathrm{d} t^{2}}(t)+\left(c d-a+\frac{b}{2}\left\|p_{n} \boldsymbol{w}(t)\right\|_{K_{n}^{2}}^{2}\right) K_{n}^{2} p_{n} \boldsymbol{w}(t)-c d U_{n}^{\prime} L_{n} p_{n} \boldsymbol{\psi}(t)+\boldsymbol{\xi}_{n}(t)=0 \\
\frac{\mathrm{d}^{2} p_{n} \boldsymbol{\psi}}{\mathrm{d} t^{2}}(t)+c L_{n}^{2} p_{n} \boldsymbol{\psi}(t)+c^{2} d\left(p_{n} \boldsymbol{\psi}(t)-L_{n} U_{n} p_{n} \boldsymbol{w}(t)\right)=0
\end{gathered}
$$

with the initial condition

$$
\frac{\mathrm{d}^{l} p_{n} \boldsymbol{w}}{\mathrm{d} t^{l}}(0)=\boldsymbol{w}_{n}^{l}, \quad \frac{\mathrm{d}^{l} p_{n} \boldsymbol{\psi}}{\mathrm{d} t^{l}}(0)=\boldsymbol{\psi}_{n}^{l}, \quad l=0,1 .
$$

In (3.7) $\boldsymbol{\xi}_{n}(t)$ is a truncation error for which the following equality holds

$$
\boldsymbol{\xi}_{n}(t)=\frac{b}{2} \pi^{2}\left(\sum_{i=n+1}^{\infty} i^{2} w_{i}^{2}(t)\right) K_{n}^{2} p_{n} \boldsymbol{w}(t)
$$

\subsection{Equations for the error}

Under the error of the method we understand the vectors $\Delta \boldsymbol{w}_{n}(t)=\boldsymbol{w}_{n}(t)-p_{n} \boldsymbol{w}(t)$ and $\Delta \boldsymbol{\psi}_{n}(t)=$ $\boldsymbol{\psi}_{n}(t)-p_{n} \boldsymbol{\psi}(t)$. To write equations for these vectors, we subtract (3.7) from (3.5), and obtain

$$
\begin{aligned}
& \frac{\mathrm{d}^{2} \Delta \boldsymbol{w}_{n}}{\mathrm{~d} t^{2}}(t)+\left(c d-a+\frac{b}{2}\left\|\boldsymbol{w}_{n}(t)\right\|_{K_{n}^{2}}^{2}\right) K_{n}^{2} \boldsymbol{w}_{n}(t) \\
& \quad-\left(c d-a+\frac{b}{2}\left\|p_{n} \boldsymbol{w}(t)\right\|_{K_{n}^{2}}^{2}\right) K_{n}^{2} p_{n} \boldsymbol{w}(t)-c d U_{n}^{\prime} L_{n} \Delta \boldsymbol{\psi}_{n}(t)=\boldsymbol{\xi}_{n}(t), \\
& \frac{\mathrm{d}^{2} \Delta \boldsymbol{\psi}_{n}}{\mathrm{~d} t^{2}}(t)+c L_{n}^{2} \Delta \boldsymbol{\psi}_{n}(t)+c^{2} d\left(\Delta \boldsymbol{\psi}_{n}(t)-L_{n} U_{n} \Delta \boldsymbol{w}_{n}(t)\right)=0 .
\end{aligned}
$$

As for the initial condition, according to (3.6) and (3.8) they can be written as

$$
\frac{\mathrm{d}^{l} \Delta \boldsymbol{w}_{n}}{\mathrm{~d} t^{l}}(0)=\frac{\mathrm{d}^{l} \boldsymbol{w}_{n}}{\mathrm{~d} t^{l}}(0)-\boldsymbol{w}_{n}^{l}, \quad \frac{\mathrm{d}^{l} \Delta \boldsymbol{\psi}_{n}}{\mathrm{~d} t^{l}}(0)=\frac{\mathrm{d}^{l} \boldsymbol{\psi}_{n}}{\mathrm{~d} t^{l}}(0)-\boldsymbol{\psi}_{n}^{l}, \quad l=0,1 .
$$


Our aim is to estimate the norms of $\Delta \boldsymbol{w}_{n}(t)$ and $\Delta \boldsymbol{\psi}_{n}(t)$. For this, we need to derive some auxiliary inequalities.

\subsection{A priori estimates}

Here we obtain the required norm estimates of the vectors which form nonlinearities in systems (3.5) and (3.7). The boundaries of these norms are calculable.

Lemma 4. The inequality

$$
\left\|p_{n} \boldsymbol{w}(t)\right\|_{K_{n}^{2}}^{2} \leq s
$$

is fulfilled, where $s$ is defined by means of the functions from the initial condition (1.2) and the parameters from the first relation of (1.4)

$$
\begin{aligned}
s=\frac{2 a}{b}+\left\{\frac { 8 } { b } \int _ { 0 } ^ { 1 } \left[\left(w^{1}(x)\right)^{2}+\right.\right. & c d\left(\psi^{0}(x)-\frac{\mathrm{d} w^{0}}{\mathrm{~d} x}(x)\right)^{2} \\
+ & \left.\left.\frac{1}{2 b}\left(-a+b \int_{0}^{1}\left(\frac{\mathrm{d} w^{0}}{\mathrm{~d} x}(x)\right)^{2} \mathrm{~d} x\right)^{2} \frac{1}{c}\left(\psi^{1}(x)\right)^{2}+\left(\frac{\mathrm{d} \psi^{0}}{\mathrm{~d} x}(x)\right)^{2}\right] \mathrm{~d} x\right\}^{\frac{1}{2}} .
\end{aligned}
$$

Proof. Multiply the equations of system (1.1) by $2 \frac{\partial w}{\partial t}(x, t)$ and $2 \frac{1}{c} \frac{\partial \psi}{\partial t}(x, t)$, respectively, integrate them with respect to $x$ from 0 to 1 and sum the obtained inequalities. Also taking into account $(1.2,1.3)$ and equality $(1.6)$, we come to the formulas

$$
\begin{aligned}
\frac{\mathrm{d}}{\mathrm{d} t} \int_{0}^{1}\left[\left(\frac{\partial w}{\partial t}(x, t)\right)^{2}+c d\left(\psi(x, t)-\frac{\partial w}{\partial x}(x, t)\right)^{2}+\frac{1}{2 b}\left(-a+b \int_{0}^{1}\left(\frac{\partial w}{\partial x}(x, t)\right)^{2} \mathrm{~d} x\right)^{2}\right. & \left.+\frac{1}{c}\left(\frac{\partial \psi}{\partial t}(x, t)\right)^{2}+\left(\frac{\partial \psi}{\partial x}(x, t)\right)^{2}\right] \mathrm{d} x=0 .
\end{aligned}
$$

This, (1.2) and also the inequality $\left\|p_{n} \boldsymbol{w}(t)\right\|_{K_{n}^{2}}^{2} \leq 2 \int_{0}^{1}\left(\frac{\partial w}{\partial t}(x, t)\right)^{2} \mathrm{~d} x$ give (3.11).

Further we will need the value $s_{n}$ defined by the vectors from the initial condition (3.6) and the prescribed parameters

$$
\begin{aligned}
s_{n}=\frac{2 a}{b}+\left\{\frac { 4 } { b } \left[\left\|\frac{\mathrm{d} \boldsymbol{w}_{n}}{\mathrm{~d} t}(0)\right\|_{n}^{2}+c d \| \boldsymbol{\psi}_{n}(0)\right.\right. & -U_{n} K_{n} \boldsymbol{w}_{n}(0) \|_{n}^{2} \\
+ & \left.\left.\frac{1}{b}\left(-a+\frac{b}{2}\left\|\boldsymbol{w}_{n}(0)\right\|_{K_{n}^{2}}^{2}\right)^{2}+\frac{1}{c}\left\|\frac{\mathrm{d} \boldsymbol{\psi}_{n}}{\mathrm{~d} t}(0)\right\|_{n}^{2}+\left\|\boldsymbol{\psi}_{n}(0)\right\|_{L_{n}^{2}}^{2}\right]\right\}^{\frac{1}{2}} .
\end{aligned}
$$

As follows from (3.2), $s_{n}$ is bounded uniformly with respect to $n$. After comparing (3.12) and (3.13), we see that in the particular case, where the initial condition (3.6) has the form

$$
\frac{\mathrm{d}^{l} \boldsymbol{w}_{n}}{\mathrm{~d} t^{l}}(0)=\boldsymbol{w}_{n}^{l}, \quad \frac{\mathrm{d}^{l} \boldsymbol{\psi}_{n}}{\mathrm{~d} t^{l}}(0)=\boldsymbol{\psi}_{n}^{l}, \quad l=0,1
$$

we have $s_{n} \rightarrow s$ as $n \rightarrow \infty$ and, moreover, $s_{n} \leq s$. 
Lemma 5. There exists a solution of problem $(3.5,3.6)$ and the estimate

$$
\left\|\boldsymbol{w}_{n}(t)\right\|_{K_{n}^{2}}^{2} \leq s_{n}
$$

is true.

Proof. Multiplying the equations of system (3.5) scalarly by $2 \frac{\mathrm{d} \boldsymbol{w}_{n}}{\mathrm{~d} t}(t)$ and $2 \frac{1}{c} \frac{\mathrm{d} \boldsymbol{\psi}_{n}}{\mathrm{~d} t}(t)$, respectively, and summing the resulting equations, we obtain the equality

$$
\frac{\mathrm{d}}{\mathrm{d} t}\left[\left\|\frac{\mathrm{d} \boldsymbol{w}_{n}}{\mathrm{~d} t}(t)\right\|_{n}^{2}+c d\left\|\boldsymbol{\psi}_{n}(t)-U_{n} K_{n} \boldsymbol{w}_{n}(t)\right\|_{n}^{2}+\frac{1}{b}\left(-a+\frac{b}{2}\left\|\boldsymbol{w}_{n}(t)\right\|_{K_{n}^{2}}^{2}\right)^{2}+\frac{1}{c}\left\|\frac{\mathrm{d} \boldsymbol{\psi}_{n}}{\mathrm{~d} t}(t)\right\|_{n}^{2}+\left\|\boldsymbol{\psi}_{n}(t)\right\|_{L_{n}^{2}}^{2}\right]=0
$$

which ensures the fulfillment of (3.14).

If, in addition, we use (3.2), then we have that the norms of the vectors $\frac{\mathrm{d}^{l} \boldsymbol{w}_{n}}{\mathrm{~d}^{l} t^{l}}(t)$ and $\frac{\mathrm{d}^{l} \boldsymbol{\psi}_{n}}{\mathrm{~d} t^{l}}(t), l=0,1$, are bounded uniformly with respect to $n$ and $t$. This fact implies the solvability of problem $(3.5,3.6)$.

\subsection{Method error}

Theorem 2. For the error of Galerkin method for $0<t \leq T$ there holds an estimate

$$
z_{n}(t) \leq C_{0} z_{n}(0)+C_{1} q^{n}
$$

where

$$
z_{n}(t)=\left\|\frac{\mathrm{d} \Delta \boldsymbol{w}_{n}}{\mathrm{~d} t}(t)\right\|_{n}+\left\|\frac{\mathrm{d} \Delta \boldsymbol{\psi}_{n}}{\mathrm{~d} t}(t)\right\|_{n}+\left\|\Delta \boldsymbol{w}_{n}(t)\right\|_{K_{n}^{2}}+\left\|\Delta \boldsymbol{\psi}_{n}(t)\right\|_{L_{n}^{2}},
$$

$C_{0}, C_{1}$ and $q<1$ are some positive constants not depending on $n$ and $t$.

Proof. Multiplying scalarly the equations of system (3.10) by $2 \frac{\mathrm{d} \Delta \boldsymbol{w}_{n}}{\mathrm{~d} t}(t)$ and $2 \frac{1}{c} \frac{\mathrm{d} \Delta \boldsymbol{\psi}_{n}}{\mathrm{~d} t}(t)$, respectively, and summing the resulting relations, we come to the equality

$$
\begin{aligned}
\frac{\mathrm{d} \Phi_{n}}{\mathrm{~d} t}(t)=\frac{b}{2}\left\|\Delta \boldsymbol{w}_{n}(t)\right\|_{K_{n}^{2}}^{2} \frac{\mathrm{d}}{\mathrm{d} t}\left\|\boldsymbol{w}_{n}(t)\right\|_{K_{n}^{2}}^{2}+b\left(\left\|p_{n} \boldsymbol{w}(t)\right\|_{K_{n}^{2}}^{2}-\left\|\boldsymbol{w}_{n}(t)\right\|_{K_{n}^{2}}^{2}\right) \\
\times\left(K_{n}^{2} p_{n} \boldsymbol{w}(t), \frac{\mathrm{d} \Delta \boldsymbol{w}_{n}}{\mathrm{~d} t}(t)\right)_{n}+2 c d \frac{\mathrm{d}}{\mathrm{d} t}\left(U_{n}^{\prime} L_{n} \Delta \boldsymbol{\psi}_{n}(t), \Delta \boldsymbol{w}_{n}(t)\right)_{n}+2\left(\boldsymbol{\xi}_{n}(t), \frac{\mathrm{d} \Delta \boldsymbol{w}_{n}}{\mathrm{~d} t}(t)\right)_{n}
\end{aligned}
$$

with the notation

$$
\begin{aligned}
\Phi_{n}(t)=\left\|\frac{\mathrm{d} \Delta \boldsymbol{w}_{n}}{\mathrm{~d} t}(t)\right\|_{n}^{2}+\frac{1}{c} \| & \frac{\mathrm{d} \Delta \boldsymbol{\psi}_{n}}{\mathrm{~d} t}(t) \|_{n}^{2} \\
& +\left(c d-a+\frac{b}{2}\left\|\boldsymbol{w}_{n}(t)\right\|_{K_{n}^{2}}^{2}\right)\left\|\Delta \boldsymbol{w}_{n}(t)\right\|_{K_{n}^{2}}^{2}+\left\|\Delta \boldsymbol{\psi}_{n}(t)\right\|_{L_{n}^{2}}^{2}+c d\left\|\Delta \boldsymbol{\psi}_{n}(t)\right\|_{n}^{2} .
\end{aligned}
$$

In our further proof we will use some constants $c_{i}>0, i=0,1, \ldots, 6$, which are expressed in terms of the initial data of the problem and the known values. This dependence, which is not quite obvious only for the first two parameters, is not given here for the sake of simplicity. A concrete definition of the parameters $c_{i}$ turns out useful when it is necessary to define the constants $C_{0}$ and $C_{1}$ from (3.15).

Let us derive some estimates. For this, we use the first formula from (3.4) and definitions (2.11) and (2.12). Note, that the uniform boundedness of the series $r_{n}(t, R)$, which was said in Corollary 3 , takes place also in the 
case where $(3.1,3.2)$ are fulfilled instead of $(2.6,2.10)$. We also apply (3.14). As a result, we have

$$
\frac{\mathrm{d}}{\mathrm{d} t}\left\|\boldsymbol{w}_{n}(t)\right\|_{K_{n}^{2}}^{2} \leq 2 \pi^{2} \sum_{i=1}^{n} i^{2}\left|w_{n i}(t)\right|\left|\frac{\mathrm{d} w_{n i}}{\mathrm{~d} t}(t)\right| \leq \pi \max \left(1, c d-a+\frac{b}{2} s_{n}\right) r_{n}(t, 1) \leq c_{0} .
$$

Again using Corollary 3 , this time its part that concerns the series $r(t, R)$, we see that

$$
\left\|K_{n}^{2} p_{n} \boldsymbol{w}(t)\right\|_{n}^{2}=\pi^{4} \sum_{i=1}^{n} i^{4} w_{i}^{2} \leq \pi^{2} i_{0} r(t, R) \leq c_{1},
$$

where $i_{0}$ is a smallest natural number such that $i<R^{i}$ holds for $i=i_{0}+1, i_{0}+2, \ldots$

By (3.20), as well as by (3.11) and (3.14), we can write

$$
\begin{aligned}
\left|\left(\left\|p_{n} \boldsymbol{w}(t)\right\|_{K_{n}^{2}}^{2}-\left\|\boldsymbol{w}_{n}(t)\right\|_{K_{n}^{2}}^{2}\right)\left(K_{n}^{2} p_{n} \boldsymbol{w}(t), \frac{\mathrm{d} \Delta \boldsymbol{w}_{n}}{\mathrm{~d} t}(t)\right)_{n}\right| \leq\left(\left\|p_{n} \boldsymbol{w}(t)\right\|_{K_{n}^{2}}\right. \\
\left.\quad+\left\|\boldsymbol{w}_{n}(t)\right\|_{K_{n}^{2}}\right)\left\|\Delta \boldsymbol{w}_{n}(t)\right\|_{K_{n}^{2}}\left\|K_{n}^{2} p_{n} \boldsymbol{w}(t)\right\|_{n}\left\|\frac{\mathrm{d} \Delta \boldsymbol{w}_{n}}{\mathrm{~d} t}(t)\right\|_{n} \leq c_{2}\left\|\Delta \boldsymbol{w}_{n}(t)\right\|_{K_{n}^{2}}\left\|\frac{\mathrm{d} \Delta \boldsymbol{w}_{n}}{\mathrm{~d} t}(t)\right\|_{n} .
\end{aligned}
$$

Furthermore, we obtain an estimate for the norm of $\boldsymbol{\xi}_{n}(t)$. Since by virtue of Theorem 1 the function $w(x, t)$ is analytic with respect to the variable $x$, there exist values $0<q<1$ and $\Omega>0$ not depending on $t$ such that $i^{2} w_{i}^{2}(t)<\Omega q^{i}$ holds for the coefficients $w_{i}(t)$ from (2.1). This fact, (3.9) and (3.20) imply

$$
\left\|\boldsymbol{\xi}_{n}(t)\right\|_{n} \leq c_{3} q^{n} .
$$

After integrating (3.17) with respect to $t$ and using estimates (3.19)-(3.22), we obtain

$$
\begin{aligned}
\Phi_{n}(t) \leq \Phi_{n}(0)+ & \int_{0}^{t}\left[\frac{b}{2} c_{0}\left\|\Delta \boldsymbol{w}_{n}(\tau)\right\|_{K_{n}^{2}}^{2}+b c_{2}\left\|\Delta \boldsymbol{w}_{n}(\tau)\right\|_{K_{n}^{2}}\left\|\frac{\mathrm{d} \Delta \boldsymbol{w}_{n}}{\mathrm{~d} \tau}(\tau)\right\|_{n}\right. \\
& \left.+2 c d\left(\left\|\frac{\mathrm{d} \Delta \boldsymbol{\psi}_{n}}{\mathrm{~d} \tau}(\tau)\right\|_{n}\left\|\Delta \boldsymbol{w}_{n}(\tau)\right\|_{K_{n}^{2}}+\left\|\Delta \boldsymbol{\psi}_{n}(\tau)\right\|_{L_{n}^{2}}\left\|\frac{\mathrm{d} \Delta \boldsymbol{w}_{n}}{\mathrm{~d} \tau}(\tau)\right\|_{n}\right)+2 c_{3} q^{n}\left\|\frac{\mathrm{d} \Delta \boldsymbol{w}_{n}}{\mathrm{~d} \tau}(\tau)\right\|_{n}\right] \mathrm{d} \tau .
\end{aligned}
$$

We transform this inequality and, using (3.16) and (3.18), replace $\Phi_{n}(t)$ by $z_{n}(t)$. As a result,

$$
z_{n}^{2}(t) \leq c_{4} z_{n}^{2}(0)+c_{5} q^{2 n}+c_{6} \int_{0}^{t} z_{n}^{2}(\tau) \mathrm{d} \tau
$$

From this we conclude by virtue of Gronwall's lemma that estimate (3.15) is valid.

\section{Approximation with Respect to the time variable}

\subsection{Transformation to a system of first order equations}

Let us write the collection of vectors

$$
\boldsymbol{u}_{n}(t), \boldsymbol{v}_{n}(t), \boldsymbol{f}_{n}(t), \boldsymbol{\varphi}_{n}(t), \boldsymbol{\psi}_{n}(t)
$$

of which the first four are the new ones defined by the formulas

$$
\boldsymbol{u}_{n}(t)=\frac{\mathrm{d} \boldsymbol{w}_{n}}{\mathrm{~d} t}(t), \boldsymbol{v}_{n}(t)=K_{n} \boldsymbol{w}_{n}(t), \boldsymbol{f}_{n}(t)=\frac{\mathrm{d} \boldsymbol{\psi}_{n}}{\mathrm{~d} t}(t), \boldsymbol{\varphi}_{n}(t)=L_{n} \boldsymbol{\psi}_{n}(t) .
$$


Applying (3.5) and (4.2), we obtain a system of equations for vectors (4.1)

$$
\begin{gathered}
\frac{\mathrm{d} \boldsymbol{u}_{n}}{\mathrm{~d} t}(t)+\left(c d-a+\frac{b}{2}\left\|\boldsymbol{v}_{n}(t)\right\|_{n}^{2}\right) K_{n} \boldsymbol{v}_{n}(t)-c d U_{n}^{\prime} \boldsymbol{\varphi}_{n}(t)=0, \\
\frac{\mathrm{d} \boldsymbol{v}_{n}}{\mathrm{~d} t}(t)=K_{n} \boldsymbol{u}_{n}(t), \frac{\mathrm{d} \boldsymbol{f}_{n}}{\mathrm{~d} t}(t)+c L_{n} \boldsymbol{\varphi}_{n}(t)+c^{2} d\left(\boldsymbol{\psi}_{n}(t)-U_{n} \boldsymbol{v}_{n}(t)\right)=0, \\
\frac{\mathrm{d} \boldsymbol{\varphi}_{n}}{\mathrm{~d} t}(t)=L_{n} \boldsymbol{f}_{n}(t), \quad \frac{\mathrm{d} \boldsymbol{\psi}_{n}}{\mathrm{~d} t}(t)=\boldsymbol{f}_{n}(t) .
\end{gathered}
$$

At the initial moment the values of vectors (4.1)

$$
\boldsymbol{u}_{n}(0), \boldsymbol{v}_{n}(0), \boldsymbol{f}_{n}(0), \boldsymbol{\varphi}_{n}(0), \boldsymbol{\psi}_{n}(0) \text { are given }
$$

by virtue of (3.6).

Thus problem $(3.5,3.6)$ is replaced by the equivalent problem $(4.3,4.4)$. After solving the latter problem, the vector $\boldsymbol{w}_{n}(t)$, which is missing in the collection (4.1), is constructed by the formula $\boldsymbol{w}_{n}(t)=K_{n}^{-1} \boldsymbol{v}_{n}(t)$.

\subsection{Scheme of Crank-Nicholson type}

Let us solve problem $(4.3,4.4)$ by the difference method. On the interval $[0, T]$, we introduce the grid $\left\{t_{m} \mid 0=t_{0}<t_{1}<\cdots<t_{M}=T\right\}$ with variable pitch $\tau_{m}=t_{m}-t_{m-1}, m=1,2, \ldots, M$. Denote the approximate values of vectors (4.1) on the $m$ th time layer, i.e. for $t=t_{m}, m=0,1, \ldots, M$, by

$$
\boldsymbol{u}_{n}^{m}, \boldsymbol{v}_{n}^{m}, \boldsymbol{f}_{n}^{m}, \boldsymbol{\varphi}_{n}^{m}, \boldsymbol{\psi}_{n}^{m} .
$$

We use the modified Crank-Nicholson scheme for $m=1,2, \ldots, M$

$$
\begin{gathered}
\frac{\boldsymbol{u}_{n}^{m}-\boldsymbol{u}_{n}^{m-1}}{\tau_{m}}+\left(c d-a+\frac{b}{2} \frac{\left\|\boldsymbol{v}_{n}^{m}\right\|_{n}^{2}+\left\|\boldsymbol{v}_{n}^{m-1}\right\|_{n}^{2}}{2}\right) K_{n} \frac{\boldsymbol{v}_{n}^{m}+\boldsymbol{v}_{n}^{m-1}}{2}-c d U_{n}^{\prime} \frac{\boldsymbol{\varphi}_{n}^{m}+\boldsymbol{\varphi}_{n}^{m-1}}{2}=0, \\
\frac{\boldsymbol{v}_{n}^{m}-\boldsymbol{v}_{n}^{m-1}}{\tau_{m}}=K_{n} \frac{\boldsymbol{u}_{n}^{m}+\boldsymbol{u}_{n}^{m-1}}{2}, \\
\frac{\boldsymbol{f}_{n}^{m}-\boldsymbol{f}_{n}^{m-1}}{\tau_{m}}+c L_{n} \frac{\boldsymbol{\varphi}_{n}^{m}+\boldsymbol{\varphi}_{n}^{m-1}}{2}+c^{2} d\left(\frac{\boldsymbol{\psi}_{n}^{m}+\boldsymbol{\psi}_{n}^{m-1}}{2}-U_{n} \frac{\boldsymbol{v}_{n}^{m}+\boldsymbol{v}_{n}^{m-1}}{2}\right)=0, \\
\frac{\boldsymbol{\varphi}_{n}^{m}-\boldsymbol{\varphi}_{n}^{m-1}}{\tau_{m}}=L_{n} \frac{\boldsymbol{f}_{n}^{m}+\boldsymbol{f}_{n}^{m-1}}{2}, \quad \frac{\boldsymbol{\psi}_{n}^{m}-\boldsymbol{\psi}_{n}^{m-1}}{\tau_{m}}=\frac{\boldsymbol{f}_{n}^{m}+\boldsymbol{f}_{n}^{m-1}}{2},
\end{gathered}
$$

assuming that the vectors

$$
\boldsymbol{u}_{n}^{0}, \boldsymbol{v}_{n}^{0}, f_{n}^{0}, \boldsymbol{\varphi}_{n}^{0}, \boldsymbol{\psi}_{n}^{0} \quad \text { are given. }
$$

(4.7) written in a more detailed form means that the vectors

$$
\begin{gathered}
\boldsymbol{u}_{n}^{0}=\boldsymbol{u}_{n}(0)+\Delta \boldsymbol{u}_{n}^{0}, \quad \boldsymbol{v}_{n}^{0}=\boldsymbol{v}_{n}(0)+\Delta \boldsymbol{v}_{n}^{0}, \quad \boldsymbol{f}_{n}^{0}=\boldsymbol{f}_{n}(0)+\Delta \boldsymbol{f}_{n}^{0}, \\
\boldsymbol{\varphi}_{n}^{0}=\boldsymbol{\varphi}_{n}(0)+\Delta \boldsymbol{\varphi}_{n}^{0}, \quad \boldsymbol{\psi}_{n}^{0}=\boldsymbol{\psi}_{n}(0)+\Delta \boldsymbol{\psi}_{n}^{0}
\end{gathered}
$$

are known, where

$$
\Delta \boldsymbol{u}_{n}^{0}, \Delta \boldsymbol{v}_{n}^{0}, \Delta \boldsymbol{f}_{n}^{0}, \Delta \boldsymbol{\varphi}_{n}^{0}, \Delta \boldsymbol{\psi}_{n}^{0}
$$

are the vectors of possible errors of the given initial values for the difference scheme.

We assume that

the norms \|\|$_{n}$ of the vectors (4.9) and $\Delta \boldsymbol{\varphi}_{n}^{0}-L_{n} \Delta \boldsymbol{\psi}_{n}^{0}$ are bounded uniformly with respect to $n$. 


\subsection{Operator notation of the system}

Define the vector

$$
\boldsymbol{y}_{n}^{m}=\left(\boldsymbol{u}_{n}^{m}, \boldsymbol{v}_{n}^{m}, \boldsymbol{f}_{n}^{m}, \boldsymbol{\varphi}_{n}^{m}, \boldsymbol{\psi}_{n}^{m}\right), \quad m=0,1, \ldots, M .
$$

For simplicity, here and in what follows the transposition sign of vectors is omitted.

Represent scheme $(4.6,4.7)$ in the form

$$
\begin{gathered}
\frac{\boldsymbol{y}_{n}^{m}-\boldsymbol{y}_{n}^{m-1}}{\tau_{m}}=\left[A_{n}+\frac{1}{2}\left(B_{n}\left(\boldsymbol{v}_{n}^{m}\right)+B_{n}\left(\boldsymbol{v}_{n}^{m-1}\right)\right)\right] \frac{\boldsymbol{y}_{n}^{m}+\boldsymbol{y}_{n}^{m-1}}{2}, \quad m=1,2, \ldots, M, \\
\boldsymbol{y}_{n}^{0} \text { is given. }
\end{gathered}
$$

$A_{n}$ is a block square matrix of fifth order, $A_{n}=\left(A_{n i j}\right)_{i, j=1}^{5}$. Nonzero blocks in this matrix are of the form

$$
\begin{gathered}
A_{n 12}=-(c d-a) K_{n}, \quad A_{n 14}=c d U_{n}^{\prime}, \quad A_{n 21}=K_{n}, \quad A_{n 32}=c^{2} d U_{n} \\
A_{n 34}=-c L_{n}, \quad A_{n 35}=-c^{2} d I_{n}, \quad A_{n 43}=L_{n}, \quad A_{n 53}=I_{n}
\end{gathered}
$$

where there is only one matrix that has been not used above, this is the unit matrix $I_{n}$.

As for the matrix $B_{n}$ depending on the vector, since we are going to use it in our further discussion, we define it for an arbitrary $n$-dimensional vector $\boldsymbol{v}$. The matrix $B_{n}(\boldsymbol{v})$ is a block square matrix of fifth order, $B_{n}(\boldsymbol{v})=\left(B_{n i j}(\boldsymbol{v})\right)_{i, j=1}^{5}$, containing only one nonzero block

$$
B_{n 12}(\boldsymbol{v})=-\frac{b}{2}\|\boldsymbol{v}\|_{n}^{2} K_{n}
$$

In the matrices $A_{n}$ and $B_{n}(\boldsymbol{v})$ every block is a rectangular matrix, the dimension of which depends on its position. Namely, if a block is located in the first or in the second row (column) of the block matrix, then the number of rows (columns) is equal to $n$, and to $n+1$ otherwise.

As for $\boldsymbol{y}_{n}^{0}$ from the initial condition (4.13), note that by virtue of (4.11) and (4.8) we have

$$
\boldsymbol{y}_{n}^{0}=\boldsymbol{y}_{n}(0)+\Delta \boldsymbol{y}_{n}^{0}
$$

where $\Delta \boldsymbol{y}_{n}^{0}$ denotes the vector with components (4.9).

\subsection{Equation for the error}

By analogy with (4.11) and using vector (4.1), let us form the vector

$$
\boldsymbol{y}_{n}(t)=\left(\boldsymbol{u}_{n}(t), \boldsymbol{v}_{n}(t), \boldsymbol{f}_{n}(t), \boldsymbol{\varphi}_{n}(t), \boldsymbol{\psi}_{n}(t)\right)
$$

and define the error of method $(4.12,4.13)$ on the $m$ th time layer, i.e. for $t=t_{m}$,

$$
\boldsymbol{z}_{n}^{m}=\left(\boldsymbol{z}_{n k}^{m}\right)_{k=1}^{5}
$$

as a difference

$$
\boldsymbol{z}_{n}^{m}=\boldsymbol{y}_{n}^{m}-\boldsymbol{y}_{n}\left(t_{m}\right), \quad m=0,1, \ldots, M .
$$

Replacing $\boldsymbol{y}_{n}^{m-l}$ in (4.12) and (4.16) by $\boldsymbol{z}_{n}^{m-l}+\boldsymbol{y}_{n}\left(t_{m-l}\right), l=0,1$, we obtain the equation

$$
\frac{\boldsymbol{z}_{n}^{m}-\boldsymbol{z}_{n}^{m-1}}{\tau_{m}}=A_{n} \frac{\boldsymbol{z}_{n}^{m}+\boldsymbol{z}_{n}^{m-1}}{2}+\boldsymbol{\alpha}_{n}^{m, m-1}+\frac{1}{4} \boldsymbol{\beta}_{n}^{m, m-1}, \quad m=1,2, \ldots, M,
$$

and the condition

$$
\boldsymbol{z}_{n}^{0}=\Delta \boldsymbol{y}_{n}^{0}
$$


In $(4.20)$ the truncation error of scheme $(4.12,4.13)$

$$
\boldsymbol{\alpha}_{n}^{m, m-1}=\left(\boldsymbol{\alpha}_{n k}^{m, m-1}\right)_{k=1}^{5}
$$

is equal to

$$
\boldsymbol{\alpha}_{n}^{m, m-1}=-\frac{\boldsymbol{y}_{n}\left(t_{m}\right)-\boldsymbol{y}_{n}\left(t_{m-1}\right)}{\tau_{m}}+\left[A_{n}+\frac{1}{2}\left(B_{n}\left(\boldsymbol{v}_{n}\left(t_{m}\right)\right)+B_{n}\left(\boldsymbol{v}_{n}\left(t_{m-1}\right)\right)\right)\right] \frac{\boldsymbol{y}_{n}\left(t_{m}\right)+\boldsymbol{y}_{n}\left(t_{m-1}\right)}{2},
$$

while the vector $\boldsymbol{\beta}_{n}^{m, m-1}$ is defined by the formula for $m=1,2, \ldots, M$

$$
\boldsymbol{\beta}_{n}^{m, m-1}=\left(B_{n}\left(\boldsymbol{v}_{n}^{m}\right)+B_{n}\left(\boldsymbol{v}_{n}^{m-1}\right)\right)\left(\boldsymbol{y}_{n}^{m}+\boldsymbol{y}_{n}^{m-1}\right)-\left(B_{n}\left(\boldsymbol{v}_{n}\left(t_{m}\right)\right)+B_{n}\left(\boldsymbol{v}_{n}\left(t_{m-1}\right)\right)\right)\left(\boldsymbol{y}_{n}\left(t_{m}\right)+\boldsymbol{y}_{n}\left(t_{m-1}\right)\right) .
$$

\subsection{Different form of the equation for the error}

Let us transform equation (4.20). For this, we have to consider its term $\boldsymbol{\beta}_{n}^{m, m-1}$. Taking into account (4.24) and the form of the nonzero block of matrix $B_{n}(4.15)$, we write the vector

$$
\boldsymbol{\beta}_{n}^{m, m-1}=\left(\boldsymbol{\beta}_{n k}^{m, m-1}\right)_{k=1}^{5},
$$

where

$$
\begin{gathered}
\boldsymbol{\beta}_{n 1}^{m, m-1}=-\frac{b}{2} \sum_{i=m-1}^{m} \sum_{\substack{j=m-1 \\
\boldsymbol{\beta}_{n k}^{m, m-1}}}^{m}\left(\| \mathbf{0}_{n}, k>1,\right.
\end{gathered}
$$

and $\mathbf{0}_{n}$ denotes the zero vectors on the spaces $R^{n+l}, l=0,1$.

We have

$$
\begin{aligned}
\left\|\boldsymbol{v}_{n}^{i}\right\|_{n}^{2} K_{n} \boldsymbol{v}_{n}^{j}-\left\|\boldsymbol{v}_{n}\left(t_{i}\right)\right\|_{n}^{2} K_{n} \boldsymbol{v}_{n}\left(t_{j}\right) & =\left\|\boldsymbol{v}_{n}\left(t_{i}\right)+\boldsymbol{z}_{n 2}^{i}\right\|_{n}^{2} K_{n}\left(\boldsymbol{v}_{n}\left(t_{j}\right)+\boldsymbol{z}_{n 2}^{j}\right)-\left\|\boldsymbol{v}_{n}\left(t_{i}\right)\right\|_{n}^{2} K_{n} \boldsymbol{v}_{n}\left(t_{j}\right) \\
& =\left\|\boldsymbol{v}_{n}\left(t_{i}\right)\right\|_{n}^{2} K_{n} \boldsymbol{z}_{n 2}^{j}+\left[2\left(\boldsymbol{v}_{n}\left(t_{i}\right), \boldsymbol{z}_{n 2}^{i}\right)_{n}+\left\|\boldsymbol{z}_{n 2}^{i}\right\|_{n}^{2}\right] K_{n}\left(\boldsymbol{v}_{n}\left(t_{j}\right)+\boldsymbol{z}_{n 2}^{j}\right) \\
& =\left\|\boldsymbol{v}_{n}\left(t_{i}\right)\right\|_{n}^{2} K_{n} \boldsymbol{z}_{n 2}^{j}+\left(\boldsymbol{v}_{n}^{i}+\boldsymbol{v}_{n}\left(t_{i}\right), \boldsymbol{z}_{n 2}^{i}\right)_{n} K_{n} \boldsymbol{v}_{n}^{j} .
\end{aligned}
$$

The substitution of (4.25)-(4.27) into (4.20) gives the equation

$$
\sum_{l=0}^{1}\left[(-1)^{l} \boldsymbol{z}_{n}^{m-l}-\frac{\tau_{m}}{2}\left(A_{n} \boldsymbol{z}_{n}^{m-l}+\frac{1}{2} \boldsymbol{\beta}_{n}^{m-l}\right)\right]=\tau_{m} \boldsymbol{\alpha}_{n}^{m, m-1}
$$

where the vector $\boldsymbol{\beta}_{n}^{m-l}=\left(\boldsymbol{\beta}_{n k}^{m-l}\right)_{k=1}^{5}$ is used, the components of which have the form

$$
\begin{aligned}
\boldsymbol{\beta}_{n 1}^{m-l} & =-\frac{b}{2} \sum_{j=0}^{1}\left[\left\|\boldsymbol{v}_{n}\left(t_{m-j}\right)\right\|_{n}^{2} K_{n} \boldsymbol{z}_{n 2}^{m-l}+\left(\boldsymbol{v}_{n}^{m-l}+\boldsymbol{v}_{n}\left(t_{m-l}\right), \boldsymbol{z}_{n 2}^{m-l}\right)_{n} K_{n} \boldsymbol{v}_{n}^{m-j}\right], \\
\boldsymbol{\beta}_{n k}^{m-l} & =\mathbf{0}_{n}, k>1 .
\end{aligned}
$$




\subsection{Auxiliary proposals}

Our aim is to estimate the error $\boldsymbol{z}_{n}^{m}$ from equation (4.28). For this, we have to derive a few formulas.

Lemma 6. If the vectors $y_{1}$ and $y_{2}$, where $y_{l}=\left(u_{l}, v_{l}, f_{l}, \varphi_{l}, \psi_{l}\right), u_{l}, v_{l} \in R^{n}, f_{l}, \varphi_{l}, \psi_{l} \in R^{n+1}, l=1,2$, satisfy the equation

$$
\frac{y_{2}-y_{1}}{\tau}=\left[A_{n}+\frac{1}{2}\left(B_{n}\left(v_{2}\right)+B_{n}\left(v_{1}\right)\right)\right] \frac{y_{2}+y_{1}}{2},
$$

$\tau>0$, then

$$
\nu_{n}\left(y_{2}\right)=\nu_{n}\left(y_{1}\right)
$$

and

$$
e_{n}^{2}\left(y_{2}\right)-e_{n}^{2}\left(y_{1}\right)=\frac{\tau}{c d}\left(\nu_{n}\left(y_{1}\right), U_{n}\left(u_{2}+u_{1}\right)\right)_{n}
$$

Here

$$
\begin{gathered}
\nu_{n}\left(y_{l}\right)=\varphi_{l}-L_{n} \psi_{l}, \\
e_{n}\left(y_{l}\right)=\frac{1}{c d}\left[\left\|u_{l}\right\|_{n}^{2}+c d\left\|\psi_{l}-U_{n} v_{l}\right\|_{n}^{2}+\frac{1}{b}\left(-a+\frac{b}{2}\left\|v_{l}\right\|_{n}^{2}\right)^{2}+\frac{1}{c}\left\|f_{l}\right\|_{n}^{2}+\left\|\varphi_{l}\right\|_{n}^{2}\right]^{\frac{1}{2}} .
\end{gathered}
$$

Proof. When written in an expanded form, equation (4.30) looks like

$$
\begin{gathered}
\frac{u_{2}-u_{1}}{\tau}=-\left(c d-a+\frac{b}{2} \frac{\left\|v_{2}\right\|_{n}^{2}+\left\|v_{1}\right\|_{n}^{2}}{2}\right) K_{n} \frac{v_{2}+v_{1}}{2}+c d U_{n}^{\prime} \frac{\varphi_{2}+\varphi_{1}}{2} \\
\frac{v_{2}-v_{1}}{\tau}=K_{n} \frac{u_{2}+u_{1}}{2} \\
\frac{f_{2}-f_{1}}{\tau}=-c L_{n} \frac{\varphi_{2}+\varphi_{1}}{2}-c^{2} d\left(\frac{\psi_{2}+\psi_{1}}{2}-U_{n} \frac{v_{2}+v_{1}}{2}\right) \\
\frac{\varphi_{2}-\varphi_{1}}{\tau}=L_{n} \frac{f_{2}+f_{1}}{2}, \quad \frac{\psi_{2}-\psi_{1}}{\tau}=\frac{f_{2}+f_{1}}{2}
\end{gathered}
$$

Multiply scalarly equations $(4.35)$ by $u_{1}+u_{2}, c d\left(v_{1}+v_{2}\right), \frac{1}{c}\left(f_{1}+f_{2}\right), \varphi_{1}+\varphi_{2}, c d\left(\psi_{1}+\psi_{2}\right)$, respectively, and sum the obtained equalities. This results in

$$
\begin{aligned}
& \sum_{l=1}^{2}(-1)^{l}\left(\left\|u_{l}\right\|_{n}^{2}+c d\left\|v_{l}\right\|_{n}^{2}+\frac{1}{c}\left\|f_{l}\right\|_{n}^{2}+\left\|\varphi_{l}\right\|_{n}^{2}+c d\left\|\psi_{l}\right\|_{n}^{2}\right) \\
& +\tau\left\{\left(-\frac{a}{2}+\frac{b}{4} \frac{\left\|v_{2}\right\|_{n}^{2}+\left\|v_{1}\right\|_{n}^{2}}{2}\right)\left(K_{n}\left(v_{2}+v_{1}\right), u_{2}+u_{1}\right)_{n}\right. \\
& \left.-\frac{c d}{2}\left[\left(U_{n}\left(v_{2}+v_{1}\right), f_{2}+f_{1}\right)_{n}+\left(U_{n}^{\prime}\left(\varphi_{2}+\varphi_{1}\right), u_{2}+u_{1}\right)_{n}\right]\right\}=0 .
\end{aligned}
$$

Consider the scalar products in (4.36). Using the second and the fifth equation in (4.35), we obtain

$$
\begin{aligned}
\left(K_{n}\left(v_{2}+v_{1}\right), u_{2}+u_{1}\right)_{n} & =\left(v_{2}+v_{1}, K_{n}\left(u_{2}+u_{1}\right)\right)_{n}=\frac{2}{\tau}\left(\left\|v_{2}\right\|_{n}^{2}-\left\|v_{1}\right\|_{n}^{2}\right), \\
\left(U_{n}\left(v_{2}+v_{1}\right), f_{2}+f_{1}\right)_{n} & =\frac{2}{\tau}\left(v_{2}+v_{1}, U_{n}^{\prime}\left(\psi_{2}-\psi_{1}\right)\right)_{n} .
\end{aligned}
$$


Next, transform the expression $\left(U_{n}^{\prime}\left(\varphi_{2}+\varphi_{1}\right), u_{2}+u_{1}\right)_{n}$. From the fourth and the fifth equation of system (4.35) we obtain the equality $\varphi_{2}-\varphi_{1}=L_{n}\left(\psi_{2}-\psi_{1}\right)$ which proves the validity of formula (4.31). Using it together with the second equation of system (4.35) and (3.4), we come to

$$
\begin{aligned}
\left(U_{n}^{\prime}\left(\varphi_{2}+\varphi_{1}\right), u_{2}+u_{1}\right)_{n}=\left(U_{n}^{\prime} L_{n}\left(\psi_{2}+\psi_{1}\right)+\right. & \left.2 U_{n}^{\prime} \nu_{n}\left(y_{1}\right), u_{2}+u_{1}\right)_{n} \\
& =2\left[\frac{1}{\tau}\left(\psi_{2}+\psi_{1}, U_{n}\left(v_{2}-v_{1}\right)\right)_{n}+\left(U_{n}^{\prime} \nu_{n}\left(y_{1}\right), u_{2}+u_{1}\right)_{n}\right] .
\end{aligned}
$$

The substitution of (4.37) and (4.38) into (4.36) results in (4.32).

To establish the properties of scheme $(4.12,4.13)$ it is important to obtain an estimate for $\left\|\boldsymbol{v}_{n}^{m}\right\|_{n}, m=$ $1,2, \ldots, M$. In this connection we introduce the quantity $s_{n}^{0}$ which is defined by means of the vectors from (4.7) and (4.9) and also by the known parameters

$$
\begin{aligned}
s_{n}^{0}=\frac{2 a}{b}+\left\{\frac { 4 } { b } \left[\left\|\boldsymbol{u}_{n}^{0}\right\|_{n}^{2}+c d\left\|\boldsymbol{\psi}_{n}^{0}-U_{n} \boldsymbol{v}_{n}^{0}\right\|_{n}^{2}\right.\right. & \frac{1}{b}\left(-a+\frac{b}{2}\left\|\boldsymbol{v}_{n}^{0}\right\|_{n}^{2}\right)^{2} \\
& \left.\left.+\frac{1}{c}\left\|\boldsymbol{f}_{n}^{0}\right\|_{n}^{2}+\left\|\boldsymbol{\varphi}_{n}^{0}\right\|_{n}^{2}\right]\right\}^{\frac{1}{2}}+\frac{2}{\sqrt{b}} c d T\left\|\Delta \boldsymbol{\varphi}_{n}^{0}-L_{n} \Delta \boldsymbol{\psi}_{n}^{0}\right\|_{n} .
\end{aligned}
$$

After comparing (4.39) with (3.13) and taking (4.2) into account, it clearly follows that if the vectors of possible errors (4.9) are equal to zero, then $s_{n}^{0}$ coincides with $s_{n}$. In the general case, by virtue of $(3.2,4.8)$ and $(4.10)$ we have the uniform boundedness of $s_{n}^{0}$ with respect to $n$.

Lemma 7. System $(4.12,4.13)$ has a solution and the following a priori estimate holds

$$
\left\|\boldsymbol{v}_{n}^{m}\right\|_{n}^{2} \leq s_{n}^{0}, \quad m=1,2, \ldots, M
$$

Proof. For every $m,(4.12)$ is inscribed in scheme (4.30). Using (4.31), we write

$$
\nu_{n}\left(\boldsymbol{y}_{n}^{m-1}\right)=\nu_{n}\left(\boldsymbol{y}_{n}^{0}\right) .
$$

Applying (4.32) and (4.41) we come to the formula

$$
e_{n}^{2}\left(\boldsymbol{y}_{n}^{m}\right)-e_{n}^{2}\left(\boldsymbol{y}_{n}^{m-1}\right)=\frac{\tau_{m}}{c d}\left(\nu_{n}\left(\boldsymbol{y}_{n}^{0}\right), U_{n}\left(\boldsymbol{u}_{n}^{m}+\boldsymbol{u}_{n}^{m-1}\right)\right)_{n},
$$

which together with (4.34) and the equality $\left\|U_{n} \boldsymbol{u}_{n}^{m-l}\right\|_{n}=\left\|\boldsymbol{u}_{n}^{m-l}\right\|_{n}, l=0,1$, following from (3.4) allows us to conclude that

$$
e_{n}^{2}\left(\boldsymbol{y}_{n}^{m}\right)-\tau_{m}\left\|\nu_{n}\left(\boldsymbol{y}_{n}^{0}\right)\right\|_{n} e_{n}\left(\boldsymbol{y}_{n}^{m}\right) \leq e_{n}^{2}\left(\boldsymbol{y}_{n}^{m-1}\right)+\tau_{m}\left\|\nu_{n}\left(\boldsymbol{y}_{n}^{0}\right)\right\|_{n} e_{n}\left(\boldsymbol{y}_{n}^{m-1}\right) .
$$

Solving this inequality with respect to $e_{n}\left(\boldsymbol{y}_{n}^{m}\right)$, we obtain $e_{n}\left(\boldsymbol{y}_{n}^{m}\right) \leq e_{n}\left(\boldsymbol{y}_{n}^{m-1}\right)+\tau_{m}\left\|\nu_{n}\left(\boldsymbol{y}_{n}^{0}\right)\right\|{ }_{n}$, which implies

$$
e_{n}\left(\boldsymbol{y}_{n}^{m}\right) \leq e_{n}\left(\boldsymbol{y}_{n}^{0}\right)+T\left\|\nu_{n}\left(\boldsymbol{y}_{n}^{0}\right)\right\|_{n}, \quad m=1,2, \ldots, M .
$$

Combining this estimate, $(4.33,4.34,4.39)$ and $(4.2,4.8)$, we find first

$$
\left\|\boldsymbol{v}_{n}^{m}\right\|_{n}^{2} \leq \frac{2 a}{b}+\frac{2}{\sqrt{b}} c d e_{n}\left(\boldsymbol{y}_{n}^{m}\right) \leq \frac{2 a}{b}+\frac{2}{\sqrt{b}} c d\left(e_{n}\left(\boldsymbol{y}_{n}^{0}\right)+T\left\|\nu_{n}\left(\boldsymbol{y}_{n}^{0}\right)\right\|_{n}\right)
$$

and then (4.40). 
As for the solvability of system $(4.12,4.13)$, it is a consequence of the uniform boundedness of the norms of vectors (4.5) with respect to $n$. The boundedness for the vector $\boldsymbol{v}_{n}^{m}$ is obtained on the basis of (4.40) and also on the basis of $(3.2,4.8)$ and (4.10). The latter statement is true for the other vectors of collection (4.5), the only thing to be done is to replace (4.40) by analogous estimates obtained from (4.42) for the norms of the corresponding vectors.

Now let us estimate the norm of the matrix $A_{n}$.

Lemma 8. The inequality

$$
\left\|A_{n}\right\|_{n} \leq \gamma_{n}
$$

is fulfilled, where

$$
\begin{gathered}
\gamma_{n}=\max ^{\frac{1}{2}}\left(\gamma_{1 n}, \gamma_{2 n}, \gamma_{3 n}\right), \quad \gamma_{1 n}=\pi^{2} n^{2}+1, \quad \gamma_{2 n}=(c d-a)^{2} \pi^{2} n^{2}+c d(c d-a) \pi n+c^{3} d \pi n+2 c^{4} d^{2} \\
\gamma_{3 n}=c^{2} \pi^{2} n^{2}+c d(c d-a) \pi n+2 c^{3} d \pi n+c^{2} d^{2} .
\end{gathered}
$$

Proof. To obtain (4.43), we need to write the block matrix $A_{n}^{\prime} A_{n}=\left(a_{n i j}\right)_{i, j=1}^{5}$. For this, we will give the kinds of nonzero blocks. By (4.14)

$$
\begin{gathered}
a_{n 11}=K_{n}^{2}, \quad a_{n 22}=(c d-a)^{2} K_{n}^{2}+c^{4} d^{2} U_{n}^{\prime} U_{n}, \quad a_{n 33}=L_{n}^{2}+I_{n}^{2}, \quad a_{n 44}=c^{2} d^{2} U_{n} U_{n}^{\prime}+c^{2} L_{n}^{2}, \quad a_{n 55}=c^{4} d^{2} I_{n}, \\
a_{n 24}=a_{n 42}^{\prime}=-c d(c d-a) K_{n} U_{n}^{\prime}-c^{3} d U_{n}^{\prime} L_{n}, \quad a_{n 25}=a_{n 52}^{\prime}=-c^{4} d^{2} U_{n}^{\prime}, \quad a_{n 45}=a_{n 54}^{\prime}=c^{3} d L_{n} .
\end{gathered}
$$

Further, recall that the Euclidean norm of an arbitrary matrix $V$ is equal to the square root of the largest eigenvalue of the matrix $V^{\prime} V$ and that any eigenvalue of an arbitrary matrix $P=\left(p_{j j}\right)_{i, j=1}^{N}$ lies in one of the Gershgorin circles

$$
\left|\lambda-p_{i i}\right| \leq \sum_{\substack{j=1 \\ j \neq i}}^{N}\left|p_{i j}\right|, \quad i=1,2, \ldots, N .
$$

We are to apply the above arguments to the matrix $A_{n}$. For this, we need (4.45) and (3.4). As a result, we obtain (4.43) and (4.44).

The following statement concerns $\alpha_{n}^{m, m-1}$, the truncation error of scheme $(4.12,4.13)$.

Lemma 9. The estimate

$$
\left\|\boldsymbol{\alpha}_{n}^{m, m-1}\right\|_{n} \leq C \tau_{m}^{2}, \quad m=1,2, \ldots, M,
$$

is valid, where $C$ is the positive constant not depending on $\tau_{m}$ as well as on $m$ and $n$.

Proof. Though for a symmetrical scheme like scheme $(4.12,4.13)$ inequality $(4.46)$ is a trivial fact, we will give a typical fragment of the proof in order to make sure that (4.46) is fulfilled without imposing any additional restrictions. Besides, the arguments below can be helpful in the case where it is required that the constant $C$ from (4.46) be defined.

So, taking into account $(4.14,4.15)$, let us consider (4.23) in terms of the components of vector (4.17) and after that pass to the scalar equalities which we subject to the required transformations. As an example, we will illustrate this by the transformation of the value $\alpha_{n 1 i}^{m, m-1}$, which is the $i$ th component of the vector $\boldsymbol{\alpha}_{n 1}^{m, m-1}$ from representation (4.22). For this, we need to write some of vectors (4.1) in a detailed form, namely, $\boldsymbol{u}_{n}(t)=$ $\left(u_{n i}(t)\right)_{i=1}^{n}, \boldsymbol{v}_{n}(t)=\left(v_{n i}(t)\right)_{i=1}^{n}, \boldsymbol{\varphi}_{n}(t)=\left(\varphi_{n j}(t)\right)_{j=0}^{n}$. Now, taking into account (3.4), we obtain

$$
\begin{aligned}
\alpha_{n 1 i}^{m, m-1}=-\frac{u_{n i}\left(t_{m}\right)-u_{n i}\left(t_{m-1}\right)}{\tau_{m}}-\left(c d-a+\frac{b}{2} \frac{\left\|\boldsymbol{v}_{n}\left(t_{m}\right)\right\|_{n}^{2}+\left\|\boldsymbol{v}_{n}\left(t_{m-1}\right)\right\|_{n}^{2}}{2}\right) \pi i \frac{v_{n i}\left(t_{m}\right)+v_{n i}\left(t_{m-1}\right)}{2} & \\
+ & c d \frac{\varphi_{n i}\left(t_{m}\right)+\varphi_{n i}\left(t_{m-1}\right)}{2} .
\end{aligned}
$$


At the point $t=t_{m-1}$ we expand into a Taylor series $u_{n i}\left(t_{m}\right)$ up to third order and $v_{n i}\left(t_{m}\right), v_{n j}^{2}\left(t_{m}\right)$, $v_{n j}^{2}\left(t_{m}\right) v_{n i}\left(t_{m}\right), \quad j=1,2, \ldots, n$, and $\varphi_{n i}\left(t_{m}\right)$ up to second order with respect to $\tau_{m}$. Let us use the corresponding equation from system (4.3). Taking into account also equalities $(3.3,3.4)$ and the transformation formulas (4.2), we come to

$$
\begin{aligned}
\alpha_{n 1 i}^{m, m-1}=-\tau_{m}^{2}\left\{\frac{1}{6} \frac{\mathrm{d}^{4} w_{n i}}{\mathrm{~d} t^{4}}+\frac{1}{4}(c d-a) \pi^{2} i^{2} \frac{\mathrm{d}^{2} w_{n i}}{\mathrm{~d} t^{2}}+\frac{1}{4} b \pi^{4} i^{2} \sum_{j=1}^{n} j^{2}\left[w _ { n i } \left(w_{n j} \frac{\mathrm{d}^{2} w_{n j}}{\mathrm{~d} t^{2}}\right.\right.\right. & \\
\left.+\left(\frac{\mathrm{d} w_{n j}}{\mathrm{~d} t}\right)^{2}\right) & \left.\left.+\frac{\mathrm{d} w_{n i}}{\mathrm{~d} t} w_{n j} \frac{\mathrm{d} w_{n j}}{\mathrm{~d} t}+\frac{1}{2} \frac{\mathrm{d}^{2} w_{n i}}{\mathrm{~d} t^{2}} w_{n j}^{2}\right]-\frac{1}{4} c d \pi i \frac{\mathrm{d}^{2} \psi_{n i}}{\mathrm{~d} t^{2}}\right\},
\end{aligned}
$$

where, for brevity, the values of the function arguments are omitted. They are in general different and have only one thing in common - all of them belong to the interval $\left[t_{m-1}, t_{m}\right]$.

The formulas for the components of the rest of the vectors $\alpha_{n k}^{m, m-1}, k=2,3, \ldots, 5$, from (4.22) have, as compared with (4.47), a simpler form because the relations by which they are defined contain only linear terms.

(4.47) and analogous relations needed for the fulfillment of (4.46) are valid if $w_{n i}(t), \psi_{n j}(t) \in C^{4}(0, T)$, $i=1,2, \ldots, n, j=0,1, \ldots, n$. Moreover, to obtain (4.46) it is necessary that the sums

$$
\sum_{i=1}^{n} i^{8-2 l}\left(\frac{\mathrm{d}^{l} w_{n i}}{\mathrm{~d} t^{l}}(t)\right)^{2}, \sum_{i=1}^{n} i^{4}\left(\frac{\mathrm{d}^{k} w_{n i}}{\mathrm{~d} t^{k}}(t)\right)^{2}, \sum_{j=0}^{n} j^{8-2 l}\left(\frac{\mathrm{d}^{l} \psi_{n j}}{\mathrm{~d} t^{l}}(t)\right)^{2}, \quad l=2,3,4, k=0,1,
$$

be bounded uniformly with respect to $n$ and $t$.

To complete the proof, it remains to take into account Corollaries 1 and 3 and the fact that they are valid also in the case where $(3.1,3.2)$ hold instead of $(2.6,2.10)$.

\subsection{Accuracy of the difference scheme}

Let us estimate the norm of error (4.19).

Theorem 3. If the grid pitch satisfies the condition

$$
\tau_{m} \leq \frac{2(1-\omega)}{\sigma_{n}}
$$

then the error of scheme $(4.12,4.13)$ is estimated by

$$
\left\|\boldsymbol{z}_{n}^{m}\right\|_{n} \leq \exp \left(\frac{\rho_{m} \sigma_{n}}{\omega} t_{m}\right)\left(\left\|\boldsymbol{z}_{n}^{0}\right\|_{n}+\frac{1}{\omega} C \sum_{l=1}^{m} \tau_{l}^{3}\right)
$$

where $m=1,2, \ldots, M, \omega$ is an arbitrary number from the interval $(0,1), \sigma_{n}=\gamma_{n}+\frac{1}{2} b \pi n\left(s_{n}+\sqrt{s_{n} s_{n}^{0}}+s_{n}^{0}\right)$, $\rho_{m}=\max _{i} \tau_{i} / \min _{i} \tau_{i}, i=1,2, \ldots, m, C$ is the constant from estimate (4.46), and the parameters $\gamma_{n}, s_{n}, s_{n}^{0}$ are defined by equalities $(3.13,4.39,4.44)$.

Proof. Using equation (4.28) and relations (4.29) for the components of the vector $\boldsymbol{\beta}_{n}^{n-l}$, and also taking into account (4.43) and (3.4), we obtain

$$
\sum_{l=0}^{1}\left[\left((-1)^{l}-\frac{\tau_{m}}{2} \gamma_{n}\right)\left\|\boldsymbol{z}_{n}^{m-l}\right\|_{n}-\frac{\tau_{m}}{4}\left\|\boldsymbol{\beta}_{n}^{m-l}\right\|_{n}\right] \leq \tau_{m}\left\|\boldsymbol{\alpha}_{n}^{m, m-1}\right\|_{n}
$$


and, moreover,

$$
\left\|\boldsymbol{\beta}_{n}^{m-l}\right\|_{n} \leq \frac{1}{2} b \pi n \sum_{p=0}^{1}\left[\left\|\boldsymbol{v}_{n}\left(t_{m-p}\right)\right\|_{n}^{2}+\left(\left\|\boldsymbol{v}_{n}^{m-l}\right\|_{n}+\left\|\boldsymbol{v}_{n}\left(t_{m-l}\right)\right\|_{n}\right)\left\|\boldsymbol{v}_{n}^{m-p}\right\|_{n}\right]\left\|\boldsymbol{z}_{n 2}^{m-l}\right\|_{n}
$$

Since by (4.18) $\boldsymbol{z}_{n 2}^{m-l}$ is one of the components of the vector $\boldsymbol{z}_{n}^{m-l}$, we have $\left\|\boldsymbol{z}_{n 2}^{m-l}\right\|_{n} \leq\left\|\boldsymbol{z}_{n}^{m-l}\right\|_{n}, l=0,1$. Let us substitute this inequality into (4.51). Further we use inequality (4.40) and estimate (3.14) which, due to the second formula from (4.2), is written in the form $\left\|\boldsymbol{v}_{n}(t)\right\|_{n}^{2} \leq s_{n}$. Thus we obtain

$$
\left\|\boldsymbol{\beta}_{n}^{m-l}\right\|_{n} \leq b \pi n\left(s_{n}+\sqrt{s_{n} s_{n}^{0}}+s_{n}^{0}\right)\left\|\boldsymbol{z}_{n}^{m-l}\right\|_{n}
$$

Using this estimate in (4.50), we get

$$
\left(1-\frac{\tau_{m} \sigma_{n}}{2}\right)\left\|\boldsymbol{z}_{n}^{m}\right\|_{n} \leq\left(1+\frac{\tau_{m} \sigma_{n}}{2}\right)\left\|\left.\boldsymbol{z}_{n}^{m-1}\right|_{n}+\tau_{m}\right\| \boldsymbol{\alpha}_{n}^{m, m-1} \|_{n} .
$$

Since (4.48) implies $1-\frac{\tau_{m} \sigma_{n}}{2} \geq \omega>0$, it follows from (4.52) and (4.46) that

$$
\left\|\boldsymbol{z}_{n}^{m}\right\|_{n} \leq\left(1+\frac{\tau_{m} \sigma_{n}}{\omega}\right) \|\left.\boldsymbol{z}_{n}^{m-1}\right|_{n}+\frac{1}{\omega} C \tau_{m}^{3}
$$

Hence

$$
\left\|\boldsymbol{z}_{n}^{m}\right\|_{n} \leq \prod_{k=1}^{m}\left(1+\frac{\tau_{k} \sigma_{n}}{\omega}\right)\left(\left\|\boldsymbol{z}_{n}^{0}\right\|_{n}+\frac{1}{\omega} C \sum_{l=1}^{m} \tau_{l}^{3}\right)
$$

By (4.53) and the inequalities

$$
\tau_{k} \leq \max _{i} \tau_{i} \leq \frac{t_{m}}{m} \rho_{m}
$$

where $k=1,2, \ldots, m, i=1,2, \ldots, m$, we conclude that estimate (4.49) is valid.

Theorem 3 immediately implies

Corollary 4. Let for each $m=1,2, \ldots, m_{0}, 1 \leq m_{0} \leq M$, the grid pitch $\tau_{m}$ satisfy inequality (4.48) and for the initial error (4.21) the inequality $\left\|\boldsymbol{z}_{n}^{0}\right\|_{n} \leq N_{0} \tau^{2}$, where $\tau=\max _{m} \tau_{m}, m=1,2, \ldots, m_{0}$, be fulfilled. Then for the error of scheme $(4.12,4.13)$ on the $m_{0}$ th time layer there holds an estimate $\left\|\boldsymbol{z}_{n}^{m_{0}}\right\|_{n} \leq N_{1} \tau^{2}$. Here $N_{0}$ and $N_{1}$ are the positive constants not depending on $\tau$.

\section{Solution OF THE DISCRETE System}

\subsection{Iteration process of the Picard type}

The last part of the considered algorithm is aimed at the solution of system $(4.12,4.13)$. Note that each equation in (4.12) contains the unknown vectors from two time layers. System (4.12, 4.13) will be solved layerwise by iteration. If it is assumed that $\boldsymbol{y}_{n}^{m-1}$ has alredy been calculated, then the problem is reduced to finding $\boldsymbol{y}_{n}^{m}$ by iteration.

Since in the conditions of an approximate algorithm the vector $\boldsymbol{y}_{n}^{m-1}$ in equation (4.12) cannot be defined exactly, we have to replace it by the vector

$$
\boldsymbol{y}_{n}^{m-1, F}=\left(\boldsymbol{u}_{n}^{m-1, F}, \boldsymbol{v}_{n}^{m-1, F}, \boldsymbol{f}_{n}^{m-1, F}, \boldsymbol{\varphi}_{n}^{m-1, F}, \boldsymbol{\psi}_{n}^{m-1, F}\right)
$$


which is the final $(F)$ iteration approximation for $\boldsymbol{y}_{n}^{m-1}$ obtained on the $(m-1)$ th layer. Therefore the vector

$$
\boldsymbol{y}_{n, R}^{m}=\left(\boldsymbol{u}_{n, R}^{m}, \boldsymbol{v}_{n, R}^{m}, \boldsymbol{f}_{n, R}^{m}, \varphi_{n, R}^{m}, \boldsymbol{\psi}_{n, R}^{m}\right),
$$

but not $\boldsymbol{y}_{n}^{m}$, is a real $(R)$ solution of the resulting equation. Thus the equation

$$
\frac{\boldsymbol{y}_{n, R}^{m}-\boldsymbol{y}_{n}^{m-1, F}}{\tau_{m}}=\left[A_{n}+\frac{1}{2}\left(B_{n}\left(\boldsymbol{v}_{n, R}^{m}\right)+B_{n}\left(\boldsymbol{v}_{n}^{m-1, F}\right)\right)\right] \frac{\boldsymbol{y}_{n, R}^{m}+\boldsymbol{y}_{n}^{m-1, F}}{2}
$$

corresponds to the $m$ th layer, $m>1$.

Since starting from the second layer the same situation takes place on all layers, it is natural in the latter equation to write

$$
\boldsymbol{y}_{n, R}^{m-1, F}=\left(\boldsymbol{u}_{n, R}^{m-1, F}, \boldsymbol{v}_{n, R}^{m-1, F}, \boldsymbol{f}_{n, R}^{m-1, F}, \boldsymbol{\varphi}_{n, R}^{m-1, F}, \boldsymbol{\psi}_{n, R}^{m-1, F}\right)
$$

instead of $\boldsymbol{y}_{n}^{m-1, F}$.

As a result, for $\boldsymbol{y}_{n, R}^{m}$ we obtain the equation

$$
\frac{\boldsymbol{y}_{n, R}^{m}-\boldsymbol{y}_{n, R}^{m-1, F}}{\tau_{m}}=\left[A_{n}+\frac{1}{2}\left(B_{n}\left(\boldsymbol{v}_{n, R}^{m}\right)+B_{n}\left(\boldsymbol{v}_{n, R}^{m-1, F}\right)\right)\right] \frac{\boldsymbol{y}_{n, R}^{m}+\boldsymbol{y}_{n, R}^{m-1, F}}{2}
$$

Let (5.4) hold for $m=1$, too. For this, it is sufficient to assume that $\boldsymbol{y}_{n, R}^{0, F}$ is the vector taken in the role of $\boldsymbol{y}_{n}^{0}$ in equation (4.12) for $m=1$. We thus do not exclude the case, where for various reasons $\boldsymbol{y}_{n, R}^{0, F} \neq \boldsymbol{y}_{n}^{0}$.

The dimensions of the components of (5.1)-(5.3) coincide with those of the respective components of the vectors $\boldsymbol{y}_{n}^{m-1}$ and $\boldsymbol{y}_{n}^{m}$.

Now to solve (5.4) we are to choose an iteration process. For this, we use the Picard type algorithm

$$
\begin{gathered}
\boldsymbol{y}_{n, R}^{m, k}=\boldsymbol{y}_{n, R}^{m-1, F}+\frac{\tau_{m}}{2}\left[A_{n}+\frac{1}{2}\left(B_{n}\left(\boldsymbol{v}_{n, R}^{m, k-1}\right)+B_{n}\left(\boldsymbol{v}_{n, R}^{m-1, F}\right)\right)\right]\left(\boldsymbol{y}_{n, R}^{m, k-1}+\boldsymbol{y}_{n, R}^{m-1, F}\right), \quad k=1,2, \ldots \\
\boldsymbol{y}_{n, R}^{m, k-l}=\left(\boldsymbol{u}_{n, R}^{m, k-l}, \boldsymbol{v}_{n, R}^{m, k-l}, \boldsymbol{f}_{n, R}^{m, k-l}, \boldsymbol{\varphi}_{n, R}^{m, k-l}, \boldsymbol{\psi}_{n, R}^{m, k-l}\right)
\end{gathered}
$$

is the $(k-l)$ th iteration approximation of the vector $\boldsymbol{y}_{n, R}^{m}, l=0,1$, on the $m$ th layer and $\boldsymbol{u}_{n, R}^{m, k-l}, \boldsymbol{v}_{n, R}^{m, k-l} \in R^{n}$, $\boldsymbol{f}_{n, R}^{m, k-l}, \boldsymbol{\varphi}_{n, R}^{m, k-l}, \boldsymbol{\psi}_{n, R}^{m, k-l} \in R^{n+1}, \boldsymbol{y}_{n, R}^{m, 0}$ is the initial approximation.

Thus the approximation to $\boldsymbol{y}_{n}^{m}$ is performed by means of the vectors $\boldsymbol{y}_{n, R}^{m, k}$. Let us study the quality of this approximation.

\subsection{Auxiliary inequalities}

We need the following property of the matrix $B_{n}$.

Lemma 10. The inequality

$$
\left\|B_{n}\left(v_{1}\right) y_{2}-B_{n}\left(v_{3}\right) y_{4}\right\|_{n} \leq \frac{1}{4} b \pi n\left[\left(\left\|v_{1}\right\|_{n}^{2}+\left\|v_{3}\right\|_{n}^{2}\right)\left\|y_{2}-y_{4}\right\|_{n}+\left(\left\|v_{1}\right\|_{n}+\left\|v_{3}\right\|_{n}\right)\left(\left\|v_{2}\right\|_{n}+\| v_{4}||_{n}\right)\left\|y_{1}-y_{3}\right\|_{n}\right]
$$

is fulfilled for $y_{l}=\left(u_{l}, v_{l}, f_{l}, \varphi_{l}, \psi_{l}\right), u_{l}, v_{l} \in R^{n}, f_{l}, \varphi_{l}, \psi_{l} \in R^{n+1}, l=1,2, \ldots, 4$. 
Proof. By virtue of (4.15) we can write

$$
\begin{aligned}
\left\|B_{n}\left(v_{1}\right) y_{2}-B_{n}\left(v_{3}\right) y_{4}\right\|_{n} & \leq \frac{1}{2} \sum_{i=1,3}\left[\left\|B_{n}\left(v_{i}\right)\left(y_{2}-y_{4}\right)\right\|_{n}+\left\|\left(B_{n}\left(v_{1}\right)-B_{n}\left(v_{3}\right)\right) y_{i+1}\right\|_{n}\right] \\
& \leq \frac{1}{4} b \sum_{i=1,3}\left[\left\|v_{i}\right\|_{n}^{2}\left\|K_{n}\left(v_{2}-v_{4}\right)\right\|_{n}+\left\|K_{n} v_{i+1}\right\|_{n} \prod_{j=0}^{1}\left(\left|\left\|v_{1}\right\|_{n}+(-1)^{j}\left\|v_{3}\right\|_{n}\right|\right)\right]
\end{aligned}
$$

Let us use in (5.8) the form of the matrix $K_{n}$ from (3.4) and the inequalities

$$
\left\|v_{2}-v_{4}\right\|_{n} \leq\left\|y_{2}-y_{4}\right\|_{n}, \quad\left|\left\|v_{1}\right\|_{n}-\left\|v_{3}\right\|_{n}\right| \leq\left\|v_{1}-v_{3}\right\|_{n} \leq\left\|y_{1}-y_{3}\right\|_{n}
$$

which follow from the definition of the vector $y_{l}, l=1,2, \ldots, 4$. As a result, we obtain (5.7).

A further reasoning concerns equation (5.4). Let us estimate the norm of the vector $\boldsymbol{v}_{n, R}^{m}$ which forms the nonlinearity. As will be seen, the estimate depends on the iteration result on the preceding $(m-1)$ th layer and also on the vector $\boldsymbol{y}_{n, R}^{0, F}$ from the 0-layer and the given parameters.

We denote

$$
\begin{aligned}
s_{n, R}^{m-1, F}= & \frac{2 a}{b}+\left\{\frac { 4 } { b } \left[\left\|\boldsymbol{u}_{n, R}^{m-1, F}\right\|_{n}^{2}+c d\left\|\boldsymbol{\psi}_{n, R}^{m-1, F}-U_{n} \boldsymbol{v}_{n, R}^{m-1, F}\right\|_{n}^{2}\right.\right. \\
& \left.\left.+\frac{1}{b}\left(-a+\frac{b}{2}\left\|\boldsymbol{v}_{n, R}^{m-1, F}\right\|_{n}^{2}\right)^{2}+\frac{1}{c}\left\|\boldsymbol{f}_{n, R}^{m-1, F}\right\|_{n}^{2}+\left\|\boldsymbol{\varphi}_{n, R}^{m-1, F}\right\|_{n}^{2}\right]\right\}^{\frac{1}{2}}+\frac{2}{\sqrt{b}} c d \tau_{m}\left\|\boldsymbol{\varphi}_{n, R}^{0, F}-L_{n} \boldsymbol{\psi}_{n, R}^{0, F}\right\|_{n} .
\end{aligned}
$$

Lemma 11. The a priori estimate

$$
\left\|\boldsymbol{v}_{n, R}^{m}\right\|_{n}^{2} \leq s_{n, R}^{m-1, F}, \quad m=1,2, \ldots, M
$$

is true.

Proof. Equation (5.4), the solvability of which will be shown below, is a particular case of equation (4.30). Hence, using (4.32), we obtain

$$
e_{n}^{2}\left(\boldsymbol{y}_{n, R}^{m}\right)-e_{n}^{2}\left(\boldsymbol{y}_{n, R}^{m-1, F}\right)=\frac{\tau_{m}}{c d}\left(\nu_{n}\left(\boldsymbol{y}_{n, R}^{m-1, F}\right), U_{n}\left(\boldsymbol{u}_{n, R}^{m}+\boldsymbol{u}_{n, R}^{m-1, F}\right)\right)_{n} .
$$

System (5.5) contains the equalities

$$
\begin{gathered}
\boldsymbol{\varphi}_{n, R}^{m, k}=\boldsymbol{\varphi}_{n, R}^{m-1, F}+\frac{\tau_{m}}{2} L_{n}\left(\boldsymbol{f}_{n, R}^{m, k}+\boldsymbol{f}_{n, R}^{m-1, F}\right) \\
\boldsymbol{\psi}_{n, R}^{m, k}=\boldsymbol{\psi}_{n, R}^{m-1, F}+\frac{\tau_{m}}{2}\left(\boldsymbol{f}_{n, R}^{m, k}+\boldsymbol{f}_{n, R}^{m-1, F}\right) .
\end{gathered}
$$

Hence it follows that $\varphi_{n, R}^{m, k}-L_{n} \boldsymbol{\psi}_{n, R}^{m, k}=\varphi_{n, R}^{m-1, F}-L_{n} \boldsymbol{\psi}_{n, R}^{m-1, F}$. Therefore, using notation (4.33), we can write $\nu_{n}\left(\boldsymbol{y}_{n, R}^{m, k}\right)=\nu_{n}\left(\boldsymbol{y}_{n, R}^{m-1, F}\right)$. By substituting the number of the last iteration on the $m$ th layer into the latter formula we get $\nu_{n}\left(\boldsymbol{y}_{n, R}^{m, F}\right)=\nu_{n}\left(\boldsymbol{y}_{n, R}^{m-1, F}\right), m=1,2, \ldots, M$. Thus we obtain the equality $\nu_{n}\left(\boldsymbol{y}_{n, R}^{m, F}\right)=\nu_{n}\left(\boldsymbol{y}_{n, R}^{0, F}\right)$, the fulfillment of which is the reason for choosing process (5.5). This equality and (5.11) give

$$
e_{n}^{2}\left(\boldsymbol{y}_{n, R}^{m}\right)-e_{n}^{2}\left(\boldsymbol{y}_{n, R}^{m-1, F}\right)=\frac{\tau_{m}}{c d}\left(\nu_{n}\left(\boldsymbol{y}_{n, R}^{0, F}\right), U_{n}\left(\boldsymbol{u}_{n, R}^{m}+\boldsymbol{u}_{n, R}^{m-1, F}\right)\right)_{n} .
$$


Taking into account the above relation, the form of the matrix $U_{n}$ from (3.4) and (4.34), we have

$$
e_{n}^{2}\left(\boldsymbol{y}_{n, R}^{m}\right)-\tau_{m}\left\|\nu_{n}\left(\boldsymbol{y}_{n, R}^{0, F}\right)\right\|_{n} e_{n}\left(\boldsymbol{y}_{n, R}^{m}\right) \leq e_{n}^{2}\left(\boldsymbol{y}_{n, R}^{m-1, F}\right)+\tau_{m}\left\|\nu_{n}\left(\boldsymbol{y}_{n, R}^{0, F}\right)\right\|_{n} e_{n}\left(\boldsymbol{y}_{n, R}^{m-1, F}\right) .
$$

This yields $e_{n}\left(\boldsymbol{y}_{n, R}^{m}\right) \leq e_{n}\left(\boldsymbol{y}_{n, R}^{m-1, F}\right)+\tau_{m}\left\|\nu_{n}\left(\boldsymbol{y}_{n, R}^{0, F}\right)\right\|_{n}, \quad m=1,2, \ldots, M$. The obtained estimate, together with (4.34), allows us to write

$$
\left\|\boldsymbol{v}_{n, R}^{m}\right\|_{n}^{2} \leq \frac{2 a}{b}+\frac{2}{\sqrt{b}} c d e_{n}\left(\boldsymbol{y}_{n, R}^{m}\right) \leq \frac{2 a}{b}+\frac{2}{\sqrt{b}} c d\left(e_{n}\left(\boldsymbol{y}_{n, R}^{m-1, F}\right)+\tau_{m}\left\|\nu_{n}\left(\boldsymbol{y}_{n, R}^{0, F}\right)\right\|_{n}\right) .
$$

This formula, notations (4.33), (4.34) and (5.9) imply (5.10).

\subsection{Iteration process error}

Let us define the error of the $k$ th approximation of process (5.5) on the $m$ th layer as the vector $\boldsymbol{z}_{n, R}^{m, k}=$ $\boldsymbol{y}_{n}^{m}-\boldsymbol{y}_{n, R}^{m, k}$ and estimate its norm. For this, we introduce two sets of values $h_{m}, \nabla \alpha m, \nabla \beta m$ and $q_{m}, \tau_{\alpha m}, \tau_{\beta m}$ for each $m$ th time layer, $m=1,2, \ldots, M$. We begin with the first triple of values $h_{m}, \nabla \alpha m, \nabla \beta m$. The first of these values depends on iteration approximations, namely, on the last approximation on the $(m-1)$ th layer and on the initial one on the $m$ th layer. The other two values depend on the initial values and the last iteration approximation on the $(m-1)$ th layer. To be more exact, let

$$
\begin{aligned}
& h_{m}=\frac{1}{2}\left[\gamma_{n}\left\|\boldsymbol{y}_{n, R}^{m, 0}+\boldsymbol{y}_{n, R}^{m-1, F}\right\|_{n}+\frac{1}{4} b \pi n\left(\left\|\boldsymbol{v}_{n, R}^{m, 0}\right\|_{n}^{2}+\|\left.\boldsymbol{v}_{n, R}^{m-1, F}\right|_{n} ^{2}\right)\left\|\boldsymbol{v}_{n, R}^{m, 0}+\boldsymbol{v}_{n, R}^{m-1, F}\right\|_{n}\right], \\
& \nabla_{\alpha m}=\frac{1}{2}\left[\gamma_{n}+\frac{1}{8} b \pi n \sum_{l=1}^{2} \sum_{p=1}^{2}\left(\left(s_{n}^{0}\right)^{\frac{1}{\tau}}+\left(s_{n, R}^{m-1, F}\right)^{\frac{1}{l}}\right)^{p-1}\left(\left(s_{n}^{0}\right)^{\frac{1}{l}}+\left\|\boldsymbol{v}_{n, R}^{m-1, F}\right\|_{n}^{\frac{2}{l}}\right)^{l-p+1}\right], \\
& \nabla_{\beta m}=\frac{1}{2}\left[\gamma_{n}+\frac{1}{8} b \pi n \sum_{l=1}^{2} \sum_{p=1}^{2}\left(\left(s_{n}^{0}\right)^{\frac{1}{l}}+\left(s_{n, R}^{m-1, F}\right)^{\frac{1}{l}}\right)^{l-p+1}\left(\left(s_{n}^{0}\right)^{\frac{1}{l}}+\left\|\boldsymbol{v}_{n, R}^{m-1, F}\right\|_{n}^{\frac{2}{l}}\right)^{p-1}\right] .
\end{aligned}
$$

As for the triple of values $q_{m}, \tau_{\alpha m}, \tau_{\beta m}$, it is defined by the inequalities

$$
\begin{gathered}
0<q_{m}<1, \\
0<\tau_{\alpha m}<\frac{1}{\nabla \beta m},
\end{gathered}
$$

$$
\begin{aligned}
\frac{\tau_{\beta m}}{2 q_{m}}\left\{\gamma_{n}+\frac{3 b \pi n}{4}\left[\frac{2}{9}\left\|\boldsymbol{v}_{n, R}^{m-1, F}\right\|_{n}^{2}+\left(\frac{1}{3}\left\|\boldsymbol{v}_{n, R}^{m-1, F}\right\|_{n}\right.\right.\right. & +\max \left(\left\|\left.\boldsymbol{y}_{n, R}^{m, 0}\right|_{n},\right\| \boldsymbol{y}_{n, R}^{m-1, F} \|_{n}\right. \\
& \left.\left.\left.\left.+\tau_{\beta m} h_{m}\right)+\left(\left\|\boldsymbol{y}_{n, R}^{m, 0}-\boldsymbol{y}_{n, R}^{m-1, F}\right\|_{n}+\tau_{\beta m} h_{m}\right) \frac{q_{m}}{1-q_{m}}\right)^{2}\right]\right\} \leq 1 .
\end{aligned}
$$

From (5.12)-(5.17) it follows that the values $h_{m}, \nabla_{\alpha m}, \nabla_{\beta m}$ and $q_{m}, \tau_{\alpha m}, \tau_{\beta m}$ can be found prior to the iteration calculation on the $m$ th layer. Further, we impose on the grid pitch the requirement that

$$
0<\tau_{m} \leq \min \left(\tau_{\alpha m}, \tau_{\beta m}\right) .
$$

Finally, we introduce one more notation. By $m_{F}$ we denote the number of iterations performed on the $m$ th layer, $m=1,2, \ldots, M$. Therefore $m_{F}$ is the number of the iteration which yields $\boldsymbol{y}_{n, R}^{m, F}$. 
Theorem 4. The following statements are true

1. If conditions (5.15)-(5.18) are fulfilled for the $m$ th layer, $1 \leq m \leq M$, then the estimate

$$
\left\|\boldsymbol{z}_{n, R}^{m, k}\right\|_{n} \leq \frac{1+\tau_{\alpha m} \nabla \alpha m}{1-\tau_{\alpha m} \nabla \beta m}\left\|\boldsymbol{y}_{n}^{m-1}-\boldsymbol{y}_{n, R}^{m-1, F}\right\|_{n}+\frac{q_{m}^{k}}{1-q_{m}}\left\|\boldsymbol{y}_{n, R}^{m, 1}-\boldsymbol{y}_{n, R}^{m, 0}\right\|_{n}
$$

holds for the error of process (5.5) on this layer.

2. If conditions (5.15)-(5.18) are fulfilled for each of the layers from the first to the mth one inclusive, $1 \leq m \leq M$, then the error of process (5.5) on the mth layer satisfies the inequality for $k=1,2, \ldots$

$$
\begin{aligned}
\left\|\boldsymbol{z}_{n, R}^{m, k}\right\|_{n} \leq\left\|\boldsymbol{y}_{n}^{0}-\boldsymbol{y}_{n, R}^{0, F}\right\|_{n} \prod_{l=1}^{m} & \frac{1+\tau_{\alpha l} \nabla \alpha l}{1-\tau_{\alpha l} \nabla \beta l} \\
& +\sum_{l=1}^{m-1}\left\|\boldsymbol{y}_{n, R}^{l, 1}-\boldsymbol{y}_{n, R}^{l, 0}\right\|_{n} \frac{q_{l}^{l_{F}}}{1-q_{l}} \prod_{p=l+1}^{m} \frac{1+\tau_{\alpha p} \nabla \alpha p}{1-\tau_{\alpha p} \nabla \beta p}+\frac{q_{m}^{k}}{1-q_{m}}\left\|\boldsymbol{y}_{n, R}^{m, 1}-\boldsymbol{y}_{n, R}^{m, 0}\right\|_{n}
\end{aligned}
$$

Remark. The method by which the conditions of this theorem are satisfied is discussed in the next subsection. Proof. We use the relation

$$
\left\|\boldsymbol{z}_{n, R}^{m, k}\right\|_{n} \leq\left\|\boldsymbol{y}_{n}^{m}-\boldsymbol{y}_{n, R}^{m}\right\|_{n}+\left\|\boldsymbol{y}_{n, R}^{m}-\boldsymbol{y}_{n, R}^{m, k}\right\|_{n},
$$

where we are to estimate each term in the right-hand part of the inequality.

For simplicity, we use the notations

$$
\boldsymbol{v}_{n, R}^{m-1}=\boldsymbol{v}_{n, R}^{m-1, F}, \quad \boldsymbol{y}_{n, R}^{m-1}=\boldsymbol{y}_{n, R}^{m-1, F} .
$$

Subtracting (5.4) from (4.12), we get

$$
\sum_{i=0}^{1}(-1)^{i} \frac{\boldsymbol{y}_{n}^{m-i}-\boldsymbol{y}_{n, R}^{m-i}}{\tau_{m}}=\frac{1}{2} \sum_{i=0}^{1}\left[A_{n}\left(\boldsymbol{y}_{n}^{m-i}-\boldsymbol{y}_{n, R}^{m-i}\right)+\frac{1}{2} \sum_{j=0}^{1}\left(B_{n}\left(\boldsymbol{v}_{n}^{m-i}\right) \boldsymbol{y}_{n}^{m-j}-B_{n}\left(\boldsymbol{v}_{n, R}^{m-i}\right) \boldsymbol{y}_{n, R}^{m-j}\right)\right]
$$

By (5.7)

$$
\begin{aligned}
\left\|B_{n}\left(\boldsymbol{v}_{n}^{m-i}\right) \boldsymbol{y}_{n}^{m-j}-B_{n}\left(\boldsymbol{v}_{n, R}^{m-i}\right) \boldsymbol{y}_{n, R}^{m-j}\right\|_{n} & \leq \frac{1}{4} b \pi n \sum_{l=0}^{1}\left[\left(\left\|\boldsymbol{v}_{n}^{m-i}\right\|_{n}^{2-l}\right.\right. \\
& \left.\left.+\left\|\boldsymbol{v}_{n, R}^{m-i}\right\|_{n}^{2-l}\right)\left(\left\|\boldsymbol{v}_{n}^{m-j}\right\|_{n}+\left\|\boldsymbol{v}_{n, R}^{m-j}\right\|_{n}\right)^{l}\left\|\boldsymbol{y}_{n}^{m-j-(i-j) l}-\boldsymbol{y}_{n, R}^{m-j-(i-j) l}\right\|_{n}\right]
\end{aligned}
$$

Hence, applying $(4.40,5.10)$ and $(5.22)$, we obtain

$$
\begin{aligned}
&\left\|B_{n}\left(\boldsymbol{v}_{n}^{m}\right) \boldsymbol{y}_{n}^{m}-B_{n}\left(\boldsymbol{v}_{n, R}^{m}\right) \boldsymbol{y}_{n, R}^{m}\right\|_{n} \leq \\
& \frac{1}{4} b \pi n \sum_{l=0}^{1}\left[\left(\left(s_{n}^{0}\right)^{1-\frac{l}{2}}+\left(s_{n, R}^{m-1, F}\right)^{1-\frac{l}{2}}\right)\left(\left(s_{n}^{0}\right)^{\frac{1}{2}}+\left(s_{n, R}^{m-1, F}\right)^{\frac{1}{2}}\right)^{l}\right]\left\|\boldsymbol{y}_{n}^{m}-\boldsymbol{y}_{n, R}^{m}\right\|_{n}, \\
&\left\|B_{n}\left(\boldsymbol{v}_{n}^{m-1}\right) \boldsymbol{y}_{n}^{m-1}-B_{n}\left(\boldsymbol{v}_{n, R}^{m-1, F}\right) \boldsymbol{y}_{n, R}^{m-1, F}\right\|_{n} \leq \frac{1}{4} b \pi n \sum_{l=0}^{1}\left[\left(\left(s_{n}^{0}\right)^{1-\frac{l}{2}}\right.\right. \\
&\left.\left.+\left\|\boldsymbol{v}_{n, R}^{m-1, F}\right\|_{n}^{2-l}\right)\left(\left(s_{n}^{0}\right)^{\frac{1}{2}}+\left\|\boldsymbol{v}_{n, R}^{m-1, F}\right\|_{n}\right)^{l}\right]\left\|\boldsymbol{y}_{n}^{m-1}-\boldsymbol{y}_{n, R}^{m-1, F}\right\|_{n}
\end{aligned}
$$


and analogous inequalities which together with estimate (4.43) are substituted into (5.23). Keeping also in mind that, by condition (5.18), $\tau_{m} \leq \tau_{\alpha m}$, where $\tau_{\alpha m}$ satisfies (5.16), for the first term from (5.21) we obtain the inequality

$$
\left\|\boldsymbol{y}_{n}^{m}-\boldsymbol{y}_{n, R}^{m}\right\|_{n} \leq \frac{1+\tau_{\alpha m} \nabla \alpha m}{1-\tau_{\alpha m} \nabla \beta m}\left\|\boldsymbol{y}_{n}^{m-1}-\boldsymbol{y}_{n, R}^{m-1, F}\right\|_{n} .
$$

Further, (5.5) implies

$$
\begin{aligned}
\boldsymbol{y}_{n, R}^{m, k+1}-\boldsymbol{y}_{n, R}^{m, k}=\frac{\tau_{m}}{2}\left[\left(A_{n}+\frac{1}{2} B_{n}\left(\boldsymbol{v}_{n, R}^{m-1, F}\right)\right)\left(\boldsymbol{y}_{n, R}^{m, k}-\boldsymbol{y}_{n, R}^{m, k-1}\right)\right. & \\
& \left.+\frac{1}{2} \sum_{l=0}^{1}(-1)^{l} B_{n}\left(\boldsymbol{v}_{n, R}^{m, k-l}\right)\left(\boldsymbol{y}_{n, R}^{m, k-l}+\boldsymbol{y}_{n, R}^{m-1, F}\right)\right], \quad k=1,2, \ldots
\end{aligned}
$$

By (5.7) we write

$$
\begin{gathered}
\left\|B_{n}\left(\boldsymbol{v}_{n, R}^{m-1, F}\right)\left(\boldsymbol{y}_{n, R}^{m, k}-\boldsymbol{y}_{n, R}^{m, k-1}\right)\right\|_{n} \leq \frac{1}{2} b \pi n\left\|\boldsymbol{v}_{n, R}^{m-1, F}\right\|_{n}^{2}\left\|\boldsymbol{y}_{n, R}^{m, k}-\boldsymbol{y}_{n, R}^{m, k-1}\right\|_{n} \\
\left\|B_{n}\left(\boldsymbol{v}_{n, R}^{m, k}\right) \boldsymbol{y}_{n, R}^{m, k}-B_{n}\left(\boldsymbol{v}_{n, R}^{m, k-1}\right) \boldsymbol{y}_{n, R}^{m, k-1}\right\|_{n} \leq \frac{1}{4} b \pi n \sum_{l=0}^{1} \sum_{i=0}^{1}\left\|\boldsymbol{v}_{n, R}^{m, k-i}\right\|_{n}^{2-l}\left(\sum_{j=0}^{1}\left\|\boldsymbol{v}_{n, R}^{m, k-j}\right\|_{n}\right)^{l}\left\|\boldsymbol{y}_{n, R}^{m, k}-\boldsymbol{y}_{n, R}^{m, k-1}\right\|_{n}, \\
\left\|\left(B_{n}\left(\boldsymbol{v}_{n, R}^{m, k}\right)-B_{n}\left(\boldsymbol{v}_{n, R}^{m, k-1}\right)\right) \boldsymbol{y}_{n, R}^{m-1, F}\right\|_{n} \leq \frac{1}{2} b \pi n \sum_{l=0}^{1}\left\|\boldsymbol{v}_{n, R}^{m, k-l}\right\|_{n}\left\|\boldsymbol{v}_{n, R}^{m-1, F}\right\|_{n}\left\|\boldsymbol{y}_{n, R}^{m, k}-\boldsymbol{y}_{n, R}^{m, k-1}\right\|_{n}
\end{gathered}
$$

From (5.6) we obtain

$$
\left\|\boldsymbol{v}_{n, R}^{m, k-l}\right\|_{n} \leq\left\|\boldsymbol{y}_{n, R}^{m, k-l}\right\|_{n}, \quad l=0,1
$$

Using (5.25)-(5.27), also (5.18) and (4.43), we get

$$
\begin{aligned}
\left\|\boldsymbol{y}_{n, R}^{m, k+1}-\boldsymbol{y}_{n, R}^{m, k}\right\|_{n} & \leq \frac{\tau_{\beta m}}{2}\left\{\gamma_{n}+\frac{1}{4} b \pi n\left[\sum_{l=0}^{2}\left\|\left.\boldsymbol{y}_{n, R}^{m, k}\right|_{n} ^{2-l}\right\| \boldsymbol{y}_{n, R}^{m, k-1} \|_{n}^{l}\right.\right. \\
& \left.\left.+\left\|\boldsymbol{v}_{n, R}^{m-1, F}\right\|_{n} \sum_{l=0}^{1}\left(\left\|\boldsymbol{y}_{n, R}^{m, k-l}\right\|_{n}+\frac{1}{2}\left\|\boldsymbol{v}_{n, R}^{m-1, F}\right\|_{n}\right)\right]\right\}\left\|\boldsymbol{y}_{n, R}^{m, k}-\boldsymbol{y}_{n, R}^{m, k-1}\right\|_{n}, \quad k=1,2, \ldots
\end{aligned}
$$

Formulas $(3.4,4.15,5.5,5.12)$ and estimates $(4.33,5.18)$ imply

$$
(1-l)\left\|\boldsymbol{y}_{n, R}^{m, 0}\right\|_{n},\left\|\boldsymbol{y}_{n, R}^{m, 1}-l \boldsymbol{y}_{n, R}^{m, 0}\right\|_{n} \leq \sigma_{l m},
$$

where

$$
\sigma_{l m}=\max \left[(1-l)\left\|\boldsymbol{y}_{n, R}^{m, 0}\right\|_{n},\left\|l \boldsymbol{y}_{n, R}^{m, 0}-\boldsymbol{y}_{n, R}^{m-1, F}\right\|_{n}+\tau_{\beta m} h_{m}\right], \quad l=0,1 .
$$

Using the parameters $\sigma_{0 m}$ and $\sigma_{1 m}$, condition (5.17) can be rewritten as

$$
\frac{\tau_{\beta m}}{2}\left[\gamma_{n}+\frac{1}{4} b \pi n \sum_{l=0}^{2}(l+1)\left\|\boldsymbol{v}_{n, R}^{m-1, F}\right\|_{n}^{2-l}\left(\sigma_{0 m}+\sigma_{1 m} \frac{q_{m}}{1-q_{m}}\right)^{l}\right] \leq q_{m} .
$$

By (5.28)-(5.30) we have

$$
\left\|\boldsymbol{y}_{n, R}^{m, 2}-\boldsymbol{y}_{n, R}^{m, 1}\right\|_{n} \leq q_{m}\left\|\boldsymbol{y}_{n, R}^{m, 1}-\boldsymbol{y}_{n, R}^{m, 0}\right\|_{n} \leq \sigma_{1 m} q_{m} .
$$


This and (5.29) imply

Now assume that the inequalities

$$
\left\|\boldsymbol{y}_{n, R}^{m, 2}\right\|_{n} \leq \sigma_{0 m}+\sigma_{1 m} q_{m}
$$

$$
\begin{gathered}
\left\|\boldsymbol{y}_{n, R}^{m, k}-\boldsymbol{y}_{n, R}^{m, k-1}\right\|_{n} \leq q_{m}\left\|\boldsymbol{y}_{n, R}^{m, k-1}-\boldsymbol{y}_{n, R}^{m, k-2}\right\|_{n} \\
\left\|\boldsymbol{y}_{n, R}^{m, k}\right\|_{n} \leq \sigma_{0 m}+\sigma_{1 m} \sum_{p=1}^{k-1} q_{m}^{p}
\end{gathered}
$$

are fulfilled for $k=2,3, \ldots, l$.

Substituting the estimate

$$
\left\|\boldsymbol{y}_{n, R}^{m, l-i}\right\|_{n} \leq \sigma_{0 m}+\sigma_{1 m} \frac{q_{m}}{1-q_{m}}, \quad i=0,1,
$$

obtained from (5.34), (5.29) and (5.15) into (5.28) for $k=l$ and using, in addition, (5.30), we have

$$
\left\|\boldsymbol{y}_{n, R}^{m, l+1}-\boldsymbol{y}_{n, R}^{m, l}\right\|_{n} \leq q_{m}\left\|\boldsymbol{y}_{n, R}^{m, l}-\boldsymbol{y}_{n, R}^{m, l-1}\right\|_{n}
$$

This inequality, (5.33) and (5.29) lead to an estimate $\left\|\boldsymbol{y}_{n, R}^{m, l+1}-\boldsymbol{y}_{n, R}^{m, l}\right\|_{n} \leq \sigma_{1 m} q_{m}^{l}$, which, together with (5.34) for $k=l$, gives

$$
\left\|\boldsymbol{y}_{n, R}^{m, l+1}\right\|_{n} \leq\left\|\boldsymbol{y}_{n, R}^{m, l}\right\|_{n}+\left\|\boldsymbol{y}_{n, R}^{m, l+1}-\boldsymbol{y}_{n, R}^{m, l}\right\|_{n} \leq \sigma_{0 m}+\sigma_{1 m} \sum_{p=1}^{l} q_{m}^{p} .
$$

By (5.35) and (5.36) we conclude that (5.33) and (5.34) are fulfilled for $k=l+1$. This fact, together with (5.31) and (5.32), means that (5.33) and (5.34) hold for arbitrary $k>1$. From (5.33) follows

$$
\left\|\boldsymbol{y}_{n, R}^{m, k}-\boldsymbol{y}_{n, R}^{m, k-1}\right\|_{n} \leq q_{m}^{k-1}\left\|\boldsymbol{y}_{n, R}^{m, 1}-\boldsymbol{y}_{n, R}^{m, 0}\right\|_{n}, \quad k=1,2, \ldots
$$

Let us verify that the sequence $\left(y_{n, R}^{m, k}\right)_{k=0}^{\infty}$ is fundamental. On the strength of (5.37), for any natural number $l>0$ we have

$$
\left\|\boldsymbol{y}_{n, R}^{m, k+l}-\boldsymbol{y}_{n, R}^{m, k}\right\|_{n} \leq \sum_{p=1}^{l}\left\|\boldsymbol{y}_{n, R}^{m, k+p}-\boldsymbol{y}_{n, R}^{m, k+p-1}\right\|_{n} \leq\left\|\boldsymbol{y}_{n, R}^{m, 1}-\boldsymbol{y}_{n, R}^{m, 0}\right\|_{n} \sum_{p=1}^{l} q_{m}^{k+p-1}
$$

Therefore

$$
\left\|\boldsymbol{y}_{n, R}^{m, k+l}-\boldsymbol{y}_{n, R}^{m, k}\right\|_{n} \leq \frac{q_{m}^{k}}{1-q_{m}}\left\|\boldsymbol{y}_{n, R}^{m, 1}-\boldsymbol{y}_{n, R}^{m, 0}\right\|_{n}, \quad k=0,1, \ldots, \quad l=1,2, \ldots .
$$

For any $l$ the right-hand side of this inequality tends to zero as $k \rightarrow \infty$. Hence the sequence $\left(y_{n, R}^{m, k}\right)_{k=0}^{\infty}$ is fundamental and has $\lim \boldsymbol{y}_{n, R}^{m, k}=\boldsymbol{y}_{n, R}^{m}$. To pass to the limit in (5.5) as $k \rightarrow \infty$, we use the continuity property of the matrices $A_{n}$ and $B_{n}$. Then it obviously follows that $\boldsymbol{y}_{n, R}^{m}$ is a solution of equation (5.4). Passing to the limit in (5.38) as $l \rightarrow \infty$, we obtain

$$
\left\|\boldsymbol{y}_{n, R}^{m}-\boldsymbol{y}_{n, R}^{m, k}\right\|_{n} \leq \frac{q_{m}^{k}}{1-q_{m}}\left\|\boldsymbol{y}_{n, R}^{m, 1}-\boldsymbol{y}_{n, R}^{m, 0}\right\|_{n}
$$

If restriction (5.18) is fulfilled, then $(5.21,5.24)$ and (5.39) imply estimate $(5.19)$.

As for inequality (5.20), to prove its validity it is sufficient to use the method of mathematical induction and formula (5.19). 
From the arguments used in the proof of Theorem 4 we can draw one more conclusion about the property of the iteration process (5.5). We formulate it as:

Remark. Let conditions (5.15)-(5.18) be fulfilled for the $m$ th layer, $1 \leq m \leq M$, and $k \rightarrow \infty$. Then $\boldsymbol{y}_{n, R}^{m, k} \rightarrow$ $\boldsymbol{y}_{n, R}^{m}$ and if, in addition, $\boldsymbol{y}_{n, R}^{m-1, F}=\boldsymbol{y}_{n}^{m-1}$, then $\boldsymbol{y}_{n, R}^{m, k} \rightarrow \boldsymbol{y}_{n}^{m}$. In both cases the convergence rate is equal to the decrease rate of geometric progression with denominator $q_{m}$.

\subsection{Fulfillment of the conditions of theorem}

In conclusion, we will present some arguments as to the fulfillment of the conditions of Theorem 4. According to (5.18), the pitch $\tau_{m}$ depends on the values of $\tau_{\alpha m}$ and $\tau_{\beta m}$. By virtue of (5.16) the first of these values is defined by the parameter $\nabla_{\beta m}$, which is calculated by (5.14) prior to the realization of the iteration process on the $m$ th layer.

As for the choice of $\tau_{\beta m}$ and $q_{m}$ satisfying conditions (5.17) and (5.15), the situation is as follows. Condition (5.17) can be represented as:

$$
\frac{P_{3}\left(\tau_{\beta m}, q_{m}\right)}{q_{m}\left(1-q_{m}\right)^{2}} \leq 0
$$

where $P_{3}\left(\tau_{\beta m}, q_{m}\right)$ is a third order polynomial with respect to $\tau_{\beta m}$ and $q_{m}$. Here two approaches can be taken: either we give the parameter $0<q_{m}<1$ defining the convergence rate on the $m$ th layer and use it to find the value of $\tau_{\beta m}$ influencing the grid pitch or vice versa. It is clear that in the first case there always exists, for any $q_{m}$, a sufficiently small $\tau_{\beta m}$ such that (5.17) is fulfilled. To find the corresponding $\tau_{\beta m}$, as follows from (5.40), we have to substitute the given value of $q_{m}$ into $P_{3}\left(\tau_{\beta m}, q_{m}\right)$ and after that to satisfy the cubic inequality $P_{3}\left(\tau_{\beta m}, q_{m}\right) \leq 0$ with respect to $\tau_{\beta m}$. This is done by means of Cardano formulas. The same formulas and (5.40) are applicable to the second case as well, where $q_{m}$ is defined by the given $\tau_{\beta m}$. However it should be kept in mind that it is not any $\tau_{\beta m}$ that an admissible value of $q_{m}$ corresponds to. When this happens, $\tau_{\beta m}$ has to be made smaller.

\section{REFERENCES}

[1] S. Bernstein, On a class of functional partial differential equations. AN SSSR, Moscow, Selected Works. Izd. 3 (1961) 323-331.

[2] M. Hirschhorn and E. Reiss, Dynamic buckling of a nonlinear Timoshenko beam. SIAM J. Appl. Math. 34 (1979) $230-301$.

[3] S. Timoshenko, Théorie des vibrations. Béranger, Paris (1947).

[4] M. Tucsnak, On an initial boundary value problem for the nonlinear Timoshenko beam. Ann. Acad. Bras. Cienc. 63 (1991) 115-125.

To access this journal online:

www.edpsciences.org 